\title{
Efficient portfolios in financial markets with proportional transaction costs
}

\author{
Luciano CAMPI * $^{*}$ Elyès JOUINI ${ }^{\dagger}$ Vincent PORTE ${ }^{\ddagger}$
}

November 4, 2012

\begin{abstract}
In this article, we characterize efficient portfolios, i.e. portfolios which are optimal for at least one rational agent, in a very general multi-currency financial market model with proportional transaction costs. In our setting, transaction costs may be random, time-dependent, have jumps and the preferences of the agents are modeled by multivariate expected utility functions. We provide a complete characterization of efficient portfolios, generalizing earlier results of Dybvig [10 and Jouini and Kallal [17. We basically show that a portfolio is efficient if and only if it is cyclically anticomonotonic with respect to at least one consistent price system that prices it. Finally, we introduce the notion of utility price of a given contingent claim as the minimal amount of a given initial portfolio allowing any agent to reach the claim by trading, and give a dual representation of it as the largest proportion of the market price necessary for all agents to reach the same expected utility level 1
\end{abstract}

Keywords: Efficient portfolios, cyclic anticomonotonicity, utility maximization, proportional transaction costs, duality, utility price.

MSC Classification (2000): 91B16, 91B28.

\section{Introduction}

In this paper we characterize efficient portfolios in a general multivariate financial market with transaction costs as in [30, 4, 3], where agents can trade in finitely many risky assets (e.g. foreign currency) facing transaction costs at each trading. Such transaction costs are proportional, time dependent, random and they may have jumps.

An efficient portfolio is a portfolio which is optimal for at least one agent, in the sense that it solves an expected utility maximization problem from terminal wealth of an agent with Von Neumann-Morgenstern preferences modeled by a concave and strictly increasing utility function. The notion of portfolio's efficiency goes back to two articles by Dybvig [9, 10, one of them with the very speaking title "Inefficient Dynamic Portfolio Strategies or How to Throw Away a Million Dollars in the Stock Market" which refers to the fact that managers choosing random or poorly diversified portfolios throw away investors' money by obtaining them less return than is justified for the amount of risk taken on. In more formal terms, this means that such portfolios are not optimal for any rational agent. More precisely, in those two papers Dybvig considered a simple frictionless complete market with finitely many and equiprobable states of the world, where the no-arbitrage condition is equivalent to the existence of a unique linear pricing rule (or equivalently, a unique risk-neutral probability measure $\mathbb{Q}$ ), and proved the following result: a consumption bundle is efficient if

*LAGA Université Paris 13 and CREST. E-mail: campi@math.univ-paris13.fr

${ }^{\dagger}$ IFD and CEREMADE, Université Paris-Dauphine, and Institut Universitaire de France.

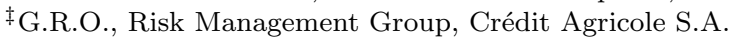

${ }^{1}$ The authors thank the "Chair Les Particuliers Face aux Risques", Fondation du Risque (Groupama-ENSAE-Dauphine), the GIP-ANR "Risk" project and the "Chair Finance and Sustainable Development" sponsored by EDF for their support. 
and only if it provides at least as much consumption in cheaper states of the world (according to the unique risk-neutral pricing rule). In mathematical terms, this can be translated as follows: a consumption bundle $X$ is efficient if and only if the two random variables $X$ and $d \mathbb{Q} / d \mathbb{P}$ are anticomonotonic, i.e. they vary in opposite directions. This characterization sheds light on the structure of efficient portfolios and gives a practical criterion to turn an inefficient claim into an efficient one, just by shuffling the claim values across the states of the world in order to get a payoff anticomonotonic to the state-price density. Many examples of this application are provided in Dybvig [10].

Relying on this characterization, Dybvig developed in [9] a new model, called payoff distribution pricing model (PDPM), and the size of inefficiency of a given claim or consumption bundle is measured as the difference between its market price and the price of the cheapest claim with the same distribution, called its "distributional price". In this approach, a claim is efficient if and only if its market price is equal to its "distributional price". This leads to a measure of portfolio performance which is independent of agents's preferences. As an application of his approach, he quantified the inefficiency size of any contingent claims, focusing on some practical example such as lock-in strategies, stop-loss strategies, rolling over portfolio insurance and so on. He found in particular that such strategies may have an inefficiency cost of the order of $0.5 \%$ per year.

In a market with frictions (e.g. with transaction costs), there are some important differences. First, the pricing rule is not linear anymore. Nonetheless, Jouini and Kallal [17] showed that the pricing rule is sublinear and can be viewed as the supremum over a set of linear pricing rules. Furthermore, in [18, they proved that Dybvig's characterization of efficient claims still holds under the following form : a portfolio is efficient if and only if it is anticomonotonic with respect to at least one pricing rule that prices it. This extension of Dybvig's characterization allowed them to compute the inefficiency size of a claim as the difference between the initial investment it requires and the largest amount needed by any rational agent to get the same utility level. They showed that it equals the difference the initial investment it requires and what they call "utility price", i.e. the minimum investment necessary to obtain a claim with the same distribution (as in Dybvig's) or a convex combination of such claims. All these claims provide to any rational agent at least as much utility as the initial claim. The utility price corresponds then to the cheapest way to guarantee to any agent at least the same utility level as with the initial claim. The inefficiency size measures then how much the claim is overpriced with respect to the utility level it provides.

The main contribution of our paper is an extension of Dybvig's characterization of efficient claims and of the notion of "utility price" to the Kabanov's multi currency market model with proportional transaction costs. Such a model can be viewed as a generalization of [17, transaction costs are random, time dependent and may have jumps. This model has been developed and studied by many authors. We refers to the recent book by Kabanov and Safarian [22] for an exhaustive treatment.

In Kabanov's setting, portfolios are vector-valued. Each entry of a given portfolio models the holding in the corresponding currency. Thus, agents' preferences are naturally described by multivariate, concave, strictly increasing (with respect to $\mathbb{R}_{+}^{d}$-preorder) utility functions. The choice of multivariate utility functions reflects the idea that the agents will not necessarily liquidate their positions to a single numeraire at the final date (which is realistic, in particular, on a currency market). This is coherent with the recent papers [1, 3] dealing with optimal investment problem under frictions. Moreover, it allows us to rely upon the duality methods developed therein. Given the intrinsic multivariate nature of the model, the crucial part to extend Dybvig's result consists in finding a suitable multivariate notion of anticomonotonicity. In convex analysis literature, one may find many definitions of multi-dimensional (anti)comonotonicity (see the complete survey by Puccetti and Scarsini [26] and the references therein). In turns out that for our purposes, the good one is the notion of cyclic anticomonotonicity as it is given in Rockafellar's book [28] (more details are given in Subsection 4.1.

Our main result can be formulated informally as follows:

Theorem 1.1 A given portfolio $X$ is efficient if and only if it is cyclically anticomonotonic with respect to some consistent price system that prices it.

This result (see Theorem 4.1 in this paper for a more precise and rigorous formulation) states essentially 
that a portfolio $X$ is efficient if and only if there exists a consistent price system $Z$ such that $X$ and $Z$ are cyclically anticomotonic. Consistent price systems are analogue of risk-neutral probability measures in multi-currency market model with transaction costs (more details are given in Section 3). This theorem considerably generalizes Dybvig [9, 10] and Jouini and Kallal [18] results.

Moreover, following the approach of Jouini and Kallal 18, we study the notion of "utility price" in this setting, which will allows us to define a measure of inefficiency that does not refer to any specific utility functions. We will provide a dual characterization of it in terms of the largest proportion of the market price of the claim needed for all every rational agents to reach the same expected utility level.

This paper is organized as follows: In Section 2 we introduce mathematical notations and definitions as well as some vector spaces that are very useful for our analysis and in particular for establishing duality results. In Section 3, we describe the financial model, recalling in particular the superhedging theorem proved in [4. Section 4 contains the main result of the paper on characterizing efficient trading strategies. Finally, Section 5 is devoted to quantifying the inefficency of a given portfolio through the notion of utility price and Section 6 concludes the paper.

\section{Definitions and useful spaces}

In this section we introduce first different notations that we will frequently use throughout the paper.

- On the space $\mathbb{R}^{d}$, we will set $\|x\|:=\max _{i}\left|x^{i}\right|$ and denote $x y$ or, equivalently, $\langle x, y\rangle$ the canonical scalar product of $x$ and $y$. The vector $\mathbf{1} \in \mathbb{R}^{d}$ is the vector whose entries are all equal to 1 . We denote by $\mathbb{R}_{+}^{d}$ the non-negative orthant, i.e. $\mathbb{R}_{+}^{d}=\left\{x \in \mathbb{R}: x_{i} \geq 0, i=1, \ldots, d\right\}$, and by $\mathbb{R}_{++}^{d}$ the positive orthant i.e., $\mathbb{R}_{++}^{d}=\left\{x \in \mathbb{R}: x_{i}>0, i=1, \ldots, d\right\}$. Given two vectors $x, y$ we will use the notation $x \geq y$ for $x-y \in \mathbb{R}_{+}^{d}, x>y$ for $x-y \in \mathbb{R}_{++}^{d}$ and $x \wedge y$ for $\left(x_{1} \wedge y_{1}, \ldots, x_{d} \wedge y_{d}\right)$.

- For a concave (utility) function $U$ defined on $\mathbb{R}_{+}^{d}$, we will denote by $\operatorname{dom} U$ its effective domain, i.e. $\operatorname{dom} U=\left\{x \in \mathbb{R}^{d}: U(x)>-\infty\right\}$ and by $\partial U$ the sub-differential of the function $-U$, i.e.

$$
\partial U(x)=\left\{y \in \mathbb{R}^{d} \mid U(z) \leq U(x)+\langle y, z-x\rangle \quad \forall z \in \mathbb{R}^{d}\right\} .
$$

- For a set $A$ we denote by $\bar{A}$ its closure and $\operatorname{int}(A)$ its interior, with respect to a given topology.

- c.d.f. will stand for cumulative distribution function.

- We denote $\chi_{A}$ the indicator function of any set $A$.

- For a random variable $X$ defined on a probability space $(\Omega, \mathcal{F}, \mathbb{P})$, we denote $\operatorname{ess} \sup X$ and $\operatorname{ess} \inf X$ the essential supremum and, respectively, the essential infimum, of the random variable $X$, i.e.

$$
\begin{aligned}
\operatorname{ess} \sup X & :=\inf \{a \in \mathbb{R}: \mathbb{P}(X>a)=0\}, \quad \inf \emptyset:=+\infty, \\
\operatorname{ess} \inf X & :=\sup \{a \in \mathbb{R}: \mathbb{P}(X<a)=0\}, \quad \sup \emptyset:=-\infty
\end{aligned}
$$

- We denote $(X)^{-}=\max (-X, 0)$ the negative part of the random variable $X$.

- Given two random vectors $X$ and $Y$, we will write $X \sim Y$ when they have the same law and we will denote by $\mathcal{L}(X)$ the set of all random vectors (defined on the same probability space) that have the same law as $X$.

Throughout the paper we will also need the following technical constructions on measures spaces.

A function $m$ from a field $\mathcal{F}$ of subsets of a set $\Omega$ to a Banach space $\mathcal{X}$ is called a finitely additive vector measure, or simply a vector measure if $m\left(A_{1} \cup A_{2}\right)=m\left(A_{1}\right)+m\left(A_{2}\right)$, whenever $A_{1}$ and $A_{2}$ are disjoint members of $\mathcal{F}$. In this paper, we will be concerned with the special case where $\mathcal{X}=\mathbb{R}^{d}$; we refer to the associated vector measure as a "Euclidean vector measure", or simply a "Euclidean measure". 
A Euclidean measure $m$ can be decomposed into its one-dimensional coordinate measures $m_{i}: \mathcal{F} \rightarrow \mathbb{R}$ by defining $m_{i}(A):=\left\langle e^{i}, m(A)\right\rangle$, where $e^{i}$ is the $i$-th canonical basis vector of $\mathbb{R}^{d}$. In this way, $m(A)=$ $\left(m_{1}(A), \ldots, m_{d}(A)\right)$ for every $A \in \mathcal{F}$. We shall say that a Euclidean measure $m$ is bounded, purely finitely additive or (weakly) absolutely continuous with respect to $\mathbb{P}$ if each of its coordinate measures is bounded, purely finitely additive or (weakly) absolutely continuous with respect to $\mathbb{P}$.

Let ba $\left(\mathbb{R}^{d}\right)=\operatorname{ba}\left(\Omega, \mathcal{F}_{T}, \mathbb{P} ; \mathbb{R}^{d}\right)$ denote the vector space of bounded Euclidean measures $m: \mathcal{F}_{T} \rightarrow \mathbb{R}^{d}$, which are (weakly) absolutely continuous with respect to $\mathbb{P}$. Let ca $\left(\mathbb{R}^{d}\right)$ the subspace of countably additive members of ba $\left(\mathbb{R}^{D}\right)$. Equipped with the norm

$$
\|m\|_{\mathrm{ba}\left(\mathbb{R}^{d}\right)}:=\sum_{i=1}^{d}\left|m_{i}\right|(\Omega),
$$

the spaces ba $\left(\mathbb{R}^{d}\right)$ and ca $\left(\mathbb{R}^{d}\right)$ are Banach spaces.

Let ba $\left(\mathbb{R}_{+}^{d}\right)$ denote the convex cone of $\mathbb{R}_{+}^{d}$-valued measures within ba $\left(\mathbb{R}^{d}\right)$. We recall the following fundamental Yosida-Hewitt decomposition: Given any $m \in \mathrm{ba}\left(\mathbb{R}^{d}\right)$ there exists a unique decomposition $m=m^{c}+m^{p}$ where $m^{c} \in \mathrm{ca}\left(\mathbb{R}^{d}\right)$ and $m^{p}$ is purely finitely additive. If $m \in \mathrm{ba}\left(\mathbb{R}_{+}^{d}\right)$ then $m^{c}, m^{p} \in \mathrm{ba}\left(\mathbb{R}_{+}^{d}\right)$.

We shall see now that elements of ba $\left(\mathbb{R}^{d}\right)$ play a natural role as linear functionals on spaces of (essentially) bounded $\mathbb{R}^{d}$-valued random variables. Let us first introduce some additional notation. Let $L^{0}\left(\mathbb{R}^{d}\right)=$ $L^{0}\left(\Omega, \mathcal{F}_{T}, \mathbb{P} ; \mathbb{R}^{d}\right)$ denote the space of $\mathbb{R}^{d}$-valued random variables (identified under the equivalence relation of a.s. equality). Given $X \in L^{0}\left(\mathbb{R}^{d}\right)$ we define the coordinate random variables $X^{i} \in L^{0}(\mathbb{R})$ for $i=1, \ldots, d$ by $X^{i}:=\left\langle X, e^{i}\right\rangle$, so that $X=\left(X^{1}, \ldots, X^{d}\right)$. Let $L^{1}\left(\mathbb{R}^{d}\right)$ denote the subspace of $L^{0}\left(\mathbb{R}^{d}\right)$ consisting of those random variables $X$ for which $\|X\|_{1}:=\mathbb{E}\left[\sum_{i}\left|X^{i}\right|\right]<\infty$. Let $L^{\infty}\left(\mathbb{R}^{d}\right)$ denote the subspace of $L^{0}\left(\mathbb{R}^{d}\right)$ consisting of those random variables $X$ for which $\|X\|_{\infty}:=\operatorname{ess} \sup \left\{\max _{i}\left|X^{i}\right|\right\}<\infty$. Finally, let $L^{\infty}\left(\mathbb{R}^{d}\right)^{*}$ denote the dual space of $\left(L^{\infty}\left(\mathbb{R}^{d}\right),\|\cdot\|_{\infty}\right)$.

We now define the map $\Psi:$ ba $\left(\mathbb{R}^{d}\right) \rightarrow L^{\infty}\left(\mathbb{R}^{d}\right)^{*}$ by

$$
(\Psi(m))(X):=\int_{\Omega}\langle X, d m\rangle:=\sum_{i=1}^{d} \int_{\Omega} X^{i} d m_{i}
$$

where $\left(m_{1}, \ldots, m_{d}\right)$ is the coordinate-wise representation of $m$. We also define the map $\Phi: \operatorname{ca}\left(\mathbb{R}^{d}\right) \rightarrow L^{1}\left(\mathbb{R}^{d}\right)$ by $\Phi(m)=\left(\frac{d m_{1}}{d \mathbb{P}}, \ldots, \frac{d m_{d}}{d \mathbb{P}}\right)$ where $\frac{d m_{i}}{d \mathbb{P}}$ is the Radon-Nikodym derivative of the $i$-th coordinate measure. Finally, we define the isometric embedding $i: L^{1}\left(\mathbb{R}^{d}\right) \rightarrow L^{\infty}\left(\mathbb{R}^{d}\right)^{*}$ by $(i(Y))(X)=\mathbb{E}[\langle X, Y\rangle]$. We recall the following two facts, whose proofs can be found in [3]:

- The maps $\Psi$ and $\Phi$ are isometric isomorphisms. Furthermore, $i \circ \Phi=\left.\Psi\right|_{\mathrm{ca}\left(\mathbb{R}^{d}\right)}$.

- $\left(\mathrm{ba}\left(\mathbb{R}^{d}\right),\|\cdot\|_{\mathrm{ba}\left(\mathbb{R}^{d}\right)}\right)$ has a $\sigma\left(\mathrm{ba}\left(\mathbb{R}^{d}\right), L^{\infty}\left(\mathbb{R}^{d}\right)\right)$-compact unit ball (Alaoglu's theorem).

For the remainder of the paper, we shall overload our notation as follows: Given $m \in$ ba $\left(\mathbb{R}^{d}\right)$ and $X \in$ $L^{\infty}\left(\mathbb{R}^{d}\right)$, we write $m(X)$ as an abbreviation of $(\Psi(m))(X)$, and we define $\frac{\mathrm{d} m}{\mathrm{~d} \mathbb{P}}:=\left(\frac{\mathrm{d} m_{1}}{\mathrm{~d} \mathbb{P}}, \ldots, \frac{\mathrm{d} m_{d}}{\mathrm{~d} \mathbb{P}}\right)=\Phi(m)$.

Given $x \in \mathbb{R}^{d}$ and $A \in \mathcal{F}_{T}$ it follows from equation 2.1) that $m\left(x \chi_{A}\right)=\langle x, m(A)\rangle$. In the special case where $A=\Omega$, we have $m(x)=\langle x, m(\Omega)\rangle$.

Let $L^{0}\left(\mathbb{R}_{+}^{d}\right)$ and $L^{\infty}\left(\mathbb{R}_{+}^{d}\right)$ denote respectively the convex cones of random variables in $L^{0}\left(\mathbb{R}^{d}\right)$ and $L^{\infty}\left(\mathbb{R}^{d}\right)$ which are $\mathbb{R}_{+}^{d}$-valued a.s.. Note that if $m \in \mathrm{ba}\left(\mathbb{R}_{+}^{d}\right)$ and $X \in L^{\infty}\left(\mathbb{R}_{+}^{d}\right)$ then $m(X) \geq 0$ (see [27, Theorem 4.4.13]). This observation allows us to extend the definition of $m(X)$ to cover the case where $m \in \operatorname{ba}\left(\mathbb{R}_{+}^{d}\right)$ and $X \in L^{0}\left(\mathbb{R}_{+}^{d}\right)$ by setting

$$
m(X):=\lim _{n \uparrow \infty} m(X \wedge(n \mathbf{1})),
$$

It is trivial that 2.2$]$ is consistent with the definition of $m(X)$ for $X \in L^{\infty}\left(\mathbb{R}^{d}\right)$. It follows that given $m_{1}, m_{2} \in \mathrm{ba}\left(\mathbb{R}_{+}^{d}\right), \lambda_{1}, \lambda_{2}, \mu_{1}, \mu_{2} \geq 0$ and $X_{1}, X_{2} \in L^{0}\left(\mathbb{R}_{+}^{d}\right)$, we have

$$
\left(\lambda_{1} m_{1}+\lambda_{2} m_{2}\right)\left(\mu_{1} X_{1}+\mu_{2} X_{2}\right)=\lambda_{1} \mu_{1} m_{1}\left(X_{1}\right)+\lambda_{1} \mu_{2} m_{1}\left(X_{2}\right)+\lambda_{2} \mu_{1} m_{2}\left(X_{1}\right)+\lambda_{2} \mu_{2} m_{2}\left(X_{2}\right) .
$$


Note that the property $i \circ \Phi=\left.\Psi\right|_{\mathrm{ca}\left(\mathbb{R}^{d}\right)}$ means that given $m \in \mathrm{ca}\left(\mathbb{R}^{d}\right)$ and $X \in L^{\infty}\left(\mathbb{R}^{d}\right)$ we have $m(X)=\mathbb{E}\left[\left\langle X, \frac{d m}{d \mathbb{P}}\right\rangle\right]$. It is easy to show that this property is also true under the extended definition 2.2 . For more details on such measures and their application to multivariate utility maximization, we refer to [3] and the references therein.

\section{The financial market}

In this section we will briefly describe the multi-currency market model where the notion of portfolios efficiency will be investigated.

\subsection{Assets and trading strategies}

Let us recall the basic features of the transaction costs model as formalized in [4 (see also [30]). In such a model, all agents can trade in $d$ assets according to a random and time varying bid-ask matrix. A $d \times d$ matrix $\Pi=\left(\pi^{i j}\right)_{1 \leq i, j \leq d}$ is called a bid-ask matrix if (i) $\pi^{i j}>0$ for every $1 \leq i, j \leq d$, (ii) $\pi^{i i}=1$ for every $1 \leq i \leq d$, and (iii) $\pi^{i j} \leq \pi^{i k} \pi^{k j}$ for every $1 \leq i, j, k \leq d$. The entry $\pi^{i j}$ represents the market exchange rate between asset $i$ and asset $j$, i.e. the number of units of assets $j$ that can be exchanged with 1 unit of asset $i$.

Given a bid-ask matrix $\Pi$, the solvency cone $K(\Pi)$ is defined as the convex polyhedral cone in $\mathbb{R}^{d}$ generated by the canonical basis vectors $e^{i}, 1 \leq i \leq d$ of $\mathbb{R}^{d}$, and the vectors $\pi^{i j} e^{i}-e^{j}, 1 \leq i, j \leq d$. The convex cone $-K(\Pi)$ should be intepreted as those portfolios available at price zero. The (positive) polar cone of $K(\Pi)$ is defined by

$$
K^{*}(\Pi)=\left\{w \in \mathbb{R}^{d}:\langle v, w\rangle \geq 0, \forall v \in K(\Pi)\right\} .
$$

The elements of $K^{*}=K^{*}(\Pi)$ can be viewed as prices (in terms of some fixed numeraire). Indeed, one can see that $w \in K^{*}$ if and only if $w \in \mathbb{R}_{+}^{d}$ and $\pi^{i j} \geq w^{j} / w^{i}$ implying that $\pi^{i j}=\left(1+\lambda^{i j}\right) w^{j} / w^{i}$ for some $\lambda^{i j}$, where $\lambda^{i j}$ can be interpreted as the proportional transaction cost and where the ratio $w^{j} / w^{i}$ can be interpreted as the frictionless exchange rate between asset $i$ and asset $j$ (see [30 for more explanations).

Next, we introduce randomness and time in our model. Let $\left(\Omega,\left(\mathcal{F}_{t}\right)_{t \in[0, T]}, \mathbb{P}\right)$ be an atomless ${ }^{1}$ filtered probability space satisfying the usual conditions and supporting all processes appearing in this paper. An adapted, càdlàg process $\left(\Pi_{t}\right)_{t \in[0, T]}$ taking values in the set of bid-ask matrices will be called a bid-ask process. A bid-ask process $\left(\Pi_{t}\right)_{t \in[0, T]}$ will now be fixed, and we drop it from the notation by writing $K_{\tau}$ (resp. $K_{\tau}^{*}$ ) instead of $K\left(\Pi_{\tau}\right)$ (resp. $K^{*}\left(\Pi_{\tau}\right)$ ) for a stopping time $\tau$.

Moreover, given any two vectors $x, y$, we will write $x \succeq_{t} y$ (resp. $x \succeq_{t}^{*} y$ ) whenever $x-y \in K_{t}$ (resp. $\left.x-y \in K_{t}^{*}\right)$.

In accordance with the framework developed in 4 we make the following technical assumption throughout the paper. The assumption is equivalent to disallowing a final trade at time $T$, but it can be relaxed via a slight modification of the model (see [4, Remark 4.2]). For this reason, we shall not explicitly mention the assumption anywhere.

Assumption $3.1 \mathcal{F}_{T-}=\mathcal{F}_{T}$ and $\Pi_{T-}=\Pi_{T}$ a.s.

Definition 3.1 An adapted, $\mathbb{R}_{+}^{d} \backslash\{0\}$-valued, càdlàg martingale $Z=\left(Z_{t}\right)_{t \in[0, T]}$ is called a consistent price process for the bid-ask process $\left(\Pi_{t}\right)_{t \in[0, T]}$ if $Z_{t} \in K_{t}^{*}$ a.s. for every $t \in[0, T]$. Moreover, $Z$ will be called $a$ strictly consistent price system if it satisfies the following additional condition: For every $[0, T] \cup\{\infty\}$-valued stopping time $\tau, Z_{\tau} \in \operatorname{int}\left(K_{\tau^{*}}\right)$ a.s. on $\{\tau<\infty\}$, and for every predictable $[0, T] \cup\{\infty\}$-valued stopping time $\sigma, Z_{\sigma-} \in \operatorname{int}\left(K_{\sigma-}^{*}\right)$ a.s. on $\{\sigma<\infty\}$. The set of all (strictly) consistent price processes will be denoted by $\mathcal{Z}\left(\mathcal{Z}^{s}\right)$.

The following assumption, which is used extensively in 4, will also hold throughout the paper.

\footnotetext{
${ }^{1}$ i.e., such a space supports a uniform random variable $U$ on $(0,1)$.
} 
Assumption 3.2 (SCPS) Existence of a strictly consistent price system: $\mathcal{Z}^{s} \neq \emptyset$.

Strictly consistent price systems are the analogue of equivalent martingale measure in financial markets with proportional transaction costs. This existence assumption is intimately related to the absence of arbitrage in such continuous time models (see also [17, 14, 13]). For a subset $A$ of $L^{0}\left(\mathbb{R}_{+}^{d}\right)$, we denote by $\overline{\operatorname{conv}}(A)$ the closure (for the a.s. convergence) of the convex hull generated by $A$.

Definition 3.2 Suppose that $\left(\Pi_{t}\right)_{t \in[0, T]}$ is a bid-ask process such that Assumption 3.2 holds true. An $\mathbb{R}^{d}$ valued process $V=\left(V_{t}\right)_{t \in[0, T]}$ is called a self-financing portfolio process for the bid-ask process $\left(\Pi_{t}\right)_{t \in[0, T]}$ if it satisfies the following properties:

1. It is predictable and a.e. path has finite variation (not necessarily right-continuous).

2. For every pair of stopping times $0 \leq \sigma \leq \tau \leq T$, we have

$$
V_{\tau}-V_{\sigma} \in-\overline{\operatorname{conv}}\left(\bigcup_{\sigma \leq t<\tau} K_{t}, 0\right) \quad \text { a.s. }
$$

A self-financing portfolio process $V$ is called admissible if it satisfies the additional property

3. There is a constant $a>0$ such that $V_{T}+a \mathbf{1} \in K_{T}$ a.s. and $\left\langle V_{\tau}+a \mathbf{1}, Z_{\tau}^{s}\right\rangle \geq 0$ a.s. for all $[0, T]$-valued stopping times $\tau$ and for every strictly consistent price system $Z^{s} \in \mathcal{Z}^{s}$.

Let $\mathcal{A}^{x}$ denote the set of all admissible, self-financing portfolio processes with initial endowment $x \in \mathbb{R}^{d}$, and let

$$
\mathcal{A}_{T}^{x}:=\left\{V_{T}: V \in \mathcal{A}^{x}\right\}
$$

be the set of all contingent claims attainable at time $T$ with initial endowment $x$. Note that $\mathcal{A}_{T}^{x}=x+\mathcal{A}_{T}^{0}$ for all $x \in \mathbb{R}^{d}$. Moreover, we denote $\mathcal{A}:=\cup_{x \in \mathbb{R}^{d}} \mathcal{A}^{x}$ the set of all admissible strategies.

In such a market with transactions costs, the value of a portfolio $V$ is not equivalent to its liquidation value at the final date $\left\langle V_{T}, S_{T}\right\rangle$, where $S$ is some price process measured in terms of some given numéraire, and it is restrictive to assume that utility functions are only functions of the liquidation value. It is more relevant to consider each agent endowed with a utility function $U \in \mathcal{U}$, where $\mathcal{U}$ is the set of functions $U: \mathbb{R}^{d} \rightarrow[-\infty, \infty)$ satisfying the following conditions :

- $U$ is upper semi-continuous,

- $\operatorname{int} \mathbb{R}_{+}^{d} \subset \operatorname{dom} U$ and $\overline{\operatorname{dom} U}=\mathbb{R}_{+}^{d}$,

- $U$ is strictly $\mathbb{R}_{+}^{d}$-increasing,

- $U$ is concave on $\mathbb{R}_{++}^{d}$.

Example 3.1 Examples of utility functions belonging to the class $\mathcal{U}$ are $U(x)=\sum_{i=1}^{d} U_{i}\left(x_{i}\right)$ where each $U_{i}$ is a one-dimensional CRRA utility function, and $U(x)=\prod_{i=1}^{d} x_{i}^{\gamma_{i}}$ with $\gamma_{i} \leq 1$ for all $i=1, \ldots, d$.

Each agent chooses an optimal strategy, depending on his utility function $U$ and his initial portfolio $x$. This optimal strategy is chosen in order to maximize the expected utility of the terminal holdings vector.

Definition 3.3 An admissible strategy $V \in \mathcal{A}$, with an initial portfolio $x \in \mathbb{R}^{d}$, is said to be efficient if and only if there exists a utility function $U \in \mathcal{U}$ such that $V_{T} \in \mathcal{A}_{T}^{x}$ is solution of:

$$
u(x)=\sup _{X \in \mathcal{A}_{T}^{x}} \mathbb{E}[U(X)]
$$

We say that a contingent claim $X$ is efficient if it is the terminal value of an efficient strategy $V$, i.e. $X=V_{T}$ for some efficient strategy $V$. 
For the convenience of the reader we present now a reformulation of [4, Theorem 4.1], giving a dual characterization of super-replicable contingent claims. We will use it to derive a dual formula for the amount of a given portfolio $x$ necessary to hedge a contingent claim $X$.

For some positive contingent claim $X \in L^{0}\left(\mathbb{R}_{+}^{d}, \mathcal{F}_{T}\right)$, let:

$$
\Gamma(X):=\left\{x \in \mathbb{R}^{d} \mid V_{T} \geq X \text { for some } V \in \mathcal{A}^{x}\right\} .
$$

The set $\Gamma(X)$ is the set of initial portfolios allowing to construct a strategy which hedges the contingent claim $X$. Before stating the super-hedging theorem we need to define one more set

$$
\mathcal{D}:=\left\{m \in \mathrm{ba}\left(\mathbb{R}^{d}\right): m(X) \leq 0 \quad \forall X \in \mathcal{C}\right\},
$$

where $\mathcal{C}:=\mathcal{A}_{T}^{0} \cap L^{\infty}\left(\mathbb{R}^{d}\right)$. With these definitions we can state the following result.

Theorem 3.1 (Super-replication) Let $x \in \mathbb{R}^{d}$ and let $X$ be an $\mathcal{F}_{T}$-measurable, $\mathbb{R}_{+}^{d}$-valued random variable. Under Assumption 3.2, the following are equivalent:

(i) $X \in \mathcal{A}_{T}^{x}$;

(ii) $\mathbb{E}\left[\left\langle X, Z_{T}\right\rangle\right] \leq\left\langle x, Z_{0}\right\rangle$, for all $Z \in \mathcal{Z}^{s}$;

(iii) $m(X) \leq\langle x, m(\Omega)\rangle$ for all $m \in \mathcal{D}$.

Proof. The equivalence between (i) and (ii) is a straightforward consequence of [4, Theorem 4.1]. Moreover, to each $Z \in \mathcal{Z}^{s}$ we can associate a countably additive measure $m \in \mathrm{ba}\left(\mathbb{R}_{+}^{d}\right)$ as follows $\frac{d m}{d \mathbb{P}}=Z_{T}^{s}$. Moreover, such a measure belongs to $\mathcal{D}$, since for any $X \in \mathcal{C}$ we have $m(X)=E\left[Z_{T} X\right] \leq E\left[Z_{T} V_{T}\right]$ for a $V_{T} \in \mathcal{A}_{T}^{0}$ and the supermartingale property of $Z V$ gives that $m(X) \leq 0$. This yields that (iii) implies (ii). To complete the proof it suffices to show that (i) implies (iii). Consider the contingent claim $X$ and decompose it as $X=\widetilde{X}+x$ where $\widetilde{X} \in \mathcal{A}_{T}^{0}$. Since $\widetilde{X}$ is not necessarily in $L^{\infty}\left(\mathbb{R}^{d}\right)$, we need to consider the sequence $\widetilde{X}_{n}:=\widetilde{X} \wedge n \mathbf{1}$, where the maximum is taken componentwise, i.e. with respect to the preorder induced by $\mathbb{R}_{+}^{d}$. The sequence clearly belongs to $\mathcal{C}$ and converges a.s. towards $\widetilde{X}$. For any $n \geq 1$ and $m \in \mathcal{D}$, by definition of the set $\mathcal{D}$ we have $m\left(\widetilde{X}_{n}\right) \leq 0$ so that passing to the limit yields

$$
m(X)=m(\tilde{X})+\langle x, m(\Omega)\rangle \leq\langle x, m(\Omega)\rangle
$$

which completes the proof.

\section{Extending Dybvig's characterization of efficient portfolios}

This section contains the main result of this paper establishing the equivalence between portfolio's efficiency and a suitable multivariate notion of anticomonotonicity, so generalizing Dybvig's [9] and Jouini and Kallal 18. results. Two are the crucial ingredients in order to extend such a characterization as it was anticipated in the Introduction: (i) a "good" dual theory for expected multivariate utility maximization problems and (ii) a suitable multivariate notion of anticomonotonicity between random vectors.

A duality theory have been established in a very general continuous time frictionless market model by Kramkov and Schachermayer [22] for univariate utility functions. In a multidimensional case, the problem was studied first by Deelstra and al. [6]. However, these articles focus on some suitable hypothesis on the utility function, as the one on the asymptotic elasticity, in order to ensure the existence of a solution in the primal problem. In our setting, we are not interested in minimal conditions guaranteeing existence since existence of optimal portfolios is part of the definition of efficient portfolios. Thus we do not need to impose regularity conditions on the utility functions such as the reasonable asymptotic elasticity condition. This is the reason why we will use instead recent results established in Campi and Owen [3], which seem to be tailor-made for our purposes.

In the following subsection, we introduce the notion of cyclic anticomonotonicity, which is the second important ingredient needed to obtain our extension of Dybvig's result. Together with its definition, we will present some of its equivalent formulations, that will reveal useful in the proof of our main result. 


\subsection{Cyclic anticomonotonicity}

In one-dimensional setting, two random variables $X, Y$ are anticomonotonic if they vary in opposite directions. The precise definition goes as follows.

Definition 4.1 Two univariate random variables $X$ and $Y$ defined on the same probability space $(\Omega, \mathcal{F}, \mathbb{P})$ are anticomonotonic if there exists $A \in \mathcal{F}$, with $\mathbb{P}(A)=1$, such that:

$$
\left(X(\omega)-X\left(\omega^{\prime}\right)\right)\left(Y(\omega)-Y\left(\omega^{\prime}\right)\right) \leq 0 \text { for every }\left(\omega, \omega^{\prime}\right) \in A \times A .
$$

In the multidimensional case, there exist several extensions depending on which properties of the onedimensional notion one would like to keep (see the survey 26]). In our case, it turns out that the "good" extension is the so-called cyclic anticomotonicity. Other multivariate extension with a more variational flavour have been recently studied and applied to vector risk measures and optimal risk sharing (see, e.g., [5, 11, 12, 29]).

Definition 4.2 Two d-dimensional random vectors $X$ and $Y$ defined on the same probability space $(\Omega, \mathcal{F}, \mathbb{P})$ are said to be cyclically anticomonotonic if and only there exists $A \in \mathcal{F}$ with $\mathbb{P}(A)=1$ such that for every $p \geq 2$ and $\left(\omega_{1}, \omega_{2}, \ldots, \omega_{p}\right) \in A^{p}$, we have:

$$
\sum_{i=1}^{p}\left\langle X\left(\omega_{i}\right), Y\left(\omega_{i}\right)-Y\left(\omega_{i+1}\right)\right\rangle \leq 0 .
$$

where we set $\omega_{p+1}=\omega_{1}$.

Rockafellar([28], Theorem 24.9) shows that the multivalued maps belonging to the subdifferential of some concave function are characterized by the cyclic anticomonotonicity. This is the property that makes cyclic anticomonotonicity the good multivariate extension in our setting. It will be clear from the proof of Theorem 4.1 .

The concept of anticomonotonicity and cyclic anticomonotonicity are in fact equivalent in the one dimensional setting, i.e. for random variables. However, this is not the case in the multidimensional framework (see Rockafellar [28, Section 24, in particular the discussion following the proof of Theorem 24.9, and the survey by Puccetti and Scarsini [26], where this notion of multivariate (anti)comonotonicity is called $c$ monotonicity).

Note that one could have defined cyclic anticomonotonicity using the notion of product probability spaces. These two concepts are in fact equivalent. This is the content of next proposition, whose proof is provided in the Appendix.

Proposition 4.1 Let $X, Y$ be two random vectors on the space $(\Omega, \mathcal{F}, \mathbb{P})$. For each $p \in \mathbb{N}^{*}$, define $\mathbb{P}^{\otimes p}$ as the usual product probability measure on the product space $\left(\Omega^{\otimes p}, \mathcal{F}^{\otimes p}\right)$. The cyclic anticomonotonicity between $X$ and $Y$ is equivalent to:

$$
\mathbb{P}^{\otimes p}\left[\sum_{i=1}^{p}\left\langle X\left(\omega_{i}\right), Y\left(\omega_{i}\right)-Y\left(\omega_{i+1}\right)\right\rangle \leq 0\right]=1
$$

for every $\left(\omega_{1}, \ldots, \omega_{p}\right) \in \Omega^{p}$ and for all $p \geq 2$, where we set $\omega_{p+1}=\omega_{1}$.

An important corollary of this proposition gives us a useful criterion to check whether two random vectors $X, Y$ are cyclically anticomonotonic:

Corollary 4.1 Let $d \in \mathbb{N}^{*}$ and let $(\Omega, \mathcal{F}, \mathbb{P})$ be a probability space. Suppose $X$ and $Y$ are two $d$-dimensional random vectors such that there aren't any positive number $\varepsilon>0$ and any finitely many sets $\Omega_{i} \in \mathcal{F}$ with $\mathbb{P}\left(\Omega_{i}\right)>0, i=1, \ldots, p$, such that

$$
\sum_{i=1}^{p}\left\langle Y\left(\omega_{i}\right), X\left(\omega_{i}\right)-X\left(\omega_{i+1}\right)\right\rangle \geq \varepsilon, \quad \forall\left(\omega_{1}, \ldots, \omega_{p}\right) \in \Omega_{1} \times \ldots \times \Omega_{p},
$$

where we set $\omega_{p+1}=\omega_{1}$. Then $X$ and $Y$ are cyclically anticomonotonic. 
In the one dimensional case, for two fixed distribution functions $F_{X}$ and $F_{Y}$ on discrete probability spaces with equiprobable states or on atomless probability spaces, we can find random variables $X$ distributed as $F_{X}$ and $Y$ distributed as $F_{Y}$ such that $X$ and $Y$ are anticomonotonic (see Hardy and al. [16]). The next result proves that this result is extendable to the multidimensional case thanks to the notion of cyclic anticomonotonicity. Its proof is provided in the Appendix. Recall that $\mathcal{L}(X)$ denotes the set of all random vectors (defined on the same probability space as $X$ ) that have the same law as $X$.

Proposition 4.2 Let $X_{0}, Y \in L^{0}\left(\mathbb{R}_{+}^{d}\right)$. Assume that the c.d.f. of $X_{0}$ is continuous. There exists a random vector $\tilde{X}_{0} \in \mathcal{L}\left(X_{0}\right)$, such that $\tilde{X}_{0}$ and $Y$ are cyclically anticomonotonic. Furthermore, $\tilde{X}_{0}$ satisfies

$$
\mathbb{E}\left[\tilde{X}_{0} Y\right]=\min \left\{\mathbb{E}[X Y]: X \in \mathcal{L}\left(X_{0}\right)\right\} .
$$

\subsection{Characterization of efficient portfolios}

We now have the good notion of multivariate anticomonotonicity for random vectors, so that we can finally turn to the problem of characterizing efficient portfolios, i.e. admissible portfolios solving

$$
u(x)=\sup _{X \in \mathcal{A}^{x}} \mathbb{E}\left[U\left(X_{T}\right)\right]
$$

for some utility function $U \in \mathcal{U}$ and some initial portfolio $x \in \mathbb{R}^{d}$. In this subsection we will at last give the precise statement of our extension of Dybvig's characterization of efficient portfolios together with its proof.

As we have already claimed, we will make use of the duality results that has been established in Campi and Owen [3. We denote by $U^{*}$ the conjugate function of $U$, i.e.

$$
U^{*}(y):=\sup _{x \in \mathbb{R}^{d}}\{U(x)-\langle x, y\rangle\}, \quad y \in \mathbb{R}^{d} .
$$

For $\mathcal{I}:=\operatorname{int}\left(-\mathcal{A}_{T}^{0} \cap \mathbb{R}^{d}\right)$, we know that for any initial portfolio in $\mathcal{I}$ the corresponding expected utility maximization problem is well-defined (see Proposition 3.1 in Campi and Owen [3]). Since $U=-\infty$ outside $\mathbb{R}_{+}^{d}$, we will consider without loss of generality only positive contingent claims $X_{0} \in L^{0}\left(\mathbb{R}_{+}^{d}\right)$.

Theorem 4.1 (Efficient contingent claims) Let $x_{0} \in \mathcal{I}$. A contingent claim $X_{0} \in L^{0}\left(\mathbb{R}_{+}^{d}\right)$ is efficient for the initial portfolio $x_{0}$ if and only if there exists a finitely additive measure $m_{0}=m_{0}^{c}+m_{0}^{p} \in \mathcal{D}$, such that:

(i) $Y_{0}:=\frac{d m_{0}^{c}}{d \mathbb{P}} \in \operatorname{int} \mathbb{R}_{+}^{d}$ a.s.,

(ii) $m_{0}^{c}\left(X_{0}\right)=\mathbb{E}\left[X_{0} Y_{0}\right]=m_{0}\left(x_{0}\right)$,

(iii) the random vectors $X_{0}$ and $Y_{0}=\frac{d m_{0}^{c}}{d \mathbb{P}}$ are cyclically anticomonotonic,

(iv) the following properties hold:

$$
\begin{array}{rll}
\operatorname{ess} \sup \left\|Y_{0}\right\|=\infty & \Rightarrow & \operatorname{essinf}\left\|X_{0}\right\|=0, \\
\operatorname{ess} \sup \left\|X_{0}\right\|<\infty & \Rightarrow & \operatorname{essinf}\left\|Y_{0}\right\|>0 .
\end{array}
$$

ProOF. Efficiency $\Rightarrow$ properties (i)-(iv). Let $X_{0}$ be an $\mathbb{R}_{+}^{d}$-valued efficient contingent claim for a utility function $U \in \mathcal{U}$ and an initial portfolio $x_{0}$. Since $x_{0} \in \operatorname{int}(\operatorname{dom}(u)), u$ being the value function corresponding to $U$, there exists a vector-valued finitely additive measure $m_{0}=m_{0}^{c}+m_{0}^{p} \in \mathcal{D}$ such that

$$
u\left(x_{0}\right)=\mathbb{E}\left[U\left(X_{0}\right)\right]=\mathbb{E}\left[U^{*}\left(\frac{d m_{0}^{c}}{d \mathbb{P}}\right)\right]+m_{0}\left(x_{0}\right),
$$


where the second equality is due to no duality gap result established in Proposition 3.5 in Campi and Owen 3]. On the other hand we also have

$$
\begin{aligned}
u\left(x_{0}\right) & =\mathbb{E}\left[U\left(X_{0}\right)-\left\langle X_{0}, \frac{d m_{0}^{c}}{d \mathbb{P}}\right\rangle+\left\langle X_{0}, \frac{d m_{0}^{c}}{d \mathbb{P}}\right\rangle\right] \\
& \leq \mathbb{E}\left[U^{*}\left(\frac{d m_{0}^{c}}{d \mathbb{P}}\right)\right]+\mathbb{E}\left[\left\langle X_{0}, \frac{d m_{0}^{c}}{d \mathbb{P}}\right\rangle\right] \\
& \leq \mathbb{E}\left[U^{*}\left(\frac{d m_{0}^{c}}{d \mathbb{P}}\right)\right]+m_{0}\left(x_{0}\right) .
\end{aligned}
$$

Because of 4.9), all those inequalities are in fact equalities, so that in particular we have $m_{0}^{c}\left(X_{0}\right)=m_{0}\left(x_{0}\right)$, which is property (ii).

Property (i) is in Proposition 3.9 in Campi and Owen 3 .

We now prove property (iii), i.e. that $\frac{d m_{0}^{c}}{d \mathbb{P}}$ and $X_{0}$ are cyclically anticomonotonic. First, set $Y_{0}:=\frac{d m_{0}^{c}}{d \mathbb{P}}$ and notice that by the definition of the dual function $U^{*}$ we have

$$
U(X)-X Y_{0}-U^{*}\left(Y_{0}\right) \leq 0
$$

for all contingent claim $X$ in $L^{0}\left(\mathbb{R}_{+}^{d}\right)$, and that by optimality $\mathbb{E}\left[U\left(X_{0}\right)-X_{0} Y_{0}-U^{*}\left(Y_{0}\right)\right]=0$. That implies $U\left(X_{0}\right)-X_{0} Y_{0}-U^{*}\left(Y_{0}\right)=0$ a.s., which is equivalent to $Y_{0}$ belonging a.s. to the subdifferential $\partial U\left(X_{0}\right)$ (Theorem 23.5(d) in Rockafellar [28]), which is in turn equivalent to cyclic anticomonotonicity (Theorem 24.8 in Rockafellar [28] as well as Theorem 4.7 in [26]).

We conclude this part of the proof showing the two properties in (iv). We prove first property (4.7). Suppose first there exist integers $i, j \in\{1, \ldots, d\}$ such that ess sup $Y_{0}^{i}=+\infty$ and ess inf $X_{0}^{j}>0$, and let's work toward a contradiction. There exists a real number $\varepsilon>0$, such that $\varepsilon \mathbf{1} \in \operatorname{dom} U$ and $X_{0}^{j}>2 \varepsilon$, a.s., which implies that $\left\|X_{0}\right\| \mathbf{1} \geq X_{0}^{j} \mathbf{1} \geq 2 \varepsilon \mathbf{1}$, where $\|x\|:=\max _{i}\left|x_{i}\right|$. Since $Y_{0} \in \partial U\left(X_{0}\right) \subset \partial U\left(\left\|X_{0}\right\| \mathbf{1}\right)$ a.s., we have

$$
U(\varepsilon \mathbf{1})-U\left(\left\|X_{0}\right\| \mathbf{1}\right) \leq\left\langle Y_{0},\left(\varepsilon-\left\|X_{0}\right\|\right) \mathbf{1}\right\rangle \leq\left\langle Y_{0},(\varepsilon-2 \varepsilon) \mathbf{1}\right\rangle=-\left\langle Y_{0}, \varepsilon \mathbf{1}\right\rangle .
$$

Since ess $\sup Y_{0}^{i}=+\infty$, we have in particular $\mathbb{P}\left(Y_{0}^{i}>n\right)>0$ for all $n \geq 0$ and all $i=1, \ldots, d$, so that we can choose (using a diagonal procedure) a partition $\left(A_{n}\right)_{n \geq 0}$ of the sample space, with $\mathbb{P}\left(A_{n}\right)>0$ for all $n \geq 0$ and such that $Y_{0}>n$ on $A_{n}$. It suffices to choose $A_{n}^{\prime}:=\left\{n<Y_{0} \leq n+1\right\}$ and drop those $A_{n}^{\prime}$ which have probability zero to occur to get the sequence $A_{n}$. Thus the previous inequality becomes

$$
U(\varepsilon \mathbf{1})-U\left(\left\|X_{0}\right\| \mathbf{1}\right) \leq-\sum_{n \geq 0}\left\langle Y_{0}(\omega), \varepsilon \mathbf{1}\right\rangle \chi_{A_{n}}(\omega)<-\sum_{n \geq 0}\langle n \mathbf{1}, \varepsilon \mathbf{1}\rangle \chi_{A_{n}}(\omega)
$$

Moreover, by monotonicity of the function $U$, we have $U\left(\left\|X_{0}\right\| \mathbf{1}\right) \geq U\left(X_{0}\right)>-\infty$ a.s. (since $X_{0}$ is efficient, thus a maximizer), so that the left hand side in (4.11) is finite (since $\varepsilon$ lies in the domain of $U$ ). On the other hand, we can always choose on the right hand side an $n$ big enough to get a contradiction.

Now, we prove property (4.8), i.e. ess sup $\left\|X_{0}\right\|<\infty \Rightarrow \operatorname{essinf}\left\|Y_{0}\right\|>0$. Indeed if ess sup $\left\|X_{0}\right\|<\infty$ one can choose two points $x^{\prime}, x^{\prime \prime} \in \mathbb{R}_{+}^{d}$ with $x^{\prime}-x^{\prime \prime} \in \operatorname{int} \mathbb{R}_{+}^{d}$ and $x^{\prime \prime} \geq X_{0}$ almost surely. Since $Y_{0} \in \partial U\left(X_{0}\right)$, we deduce that

$$
U\left(x^{\prime}\right)-U\left(x^{\prime \prime}\right) \leq U\left(x^{\prime}\right)-U\left(X_{0}\right) \leq\left\langle Y_{0}, x^{\prime}-X_{0}\right\rangle \leq\left\langle Y_{0}, x^{\prime}\right\rangle .
$$

Assume, by contradiction, that ess $\inf \left\|Y_{0}\right\|=0$, so that in particular we have $\mathbb{P}\left(Y_{0}^{i} \leq 1 / n\right)>0$ for all $n \geq 1$ and all $i=1, \ldots, d$. Thus consider the sequence $B_{n+1}=\left\{1 /(n+1) \leq\left\|Y_{0}\right\|<1 / n\right\}, n \geq 1$, of the sample space and assume $\mathbb{P}\left(B_{n}\right)>0$ for all $n \geq 1$ without loss of generality. Otherwise, it suffices to drop those events $B_{n}$ which have probability zero. The previous inequality gives

$$
U\left(x^{\prime}\right)-U\left(x^{\prime \prime}\right)<\sum_{n \geq 1}\left\langle\frac{1}{n} \mathbf{1}, x^{\prime} \mathbf{1}\right\rangle \chi_{B_{n}},
$$


which clearly leads to a contradiction since the left hand side is a strictly positive real number, while the right hand side can be made strictly smaller than this number on a set with strictly positive probability.

Properties (i)-(iv) $\Rightarrow$ efficiency. Let $X_{0}$ and $m_{0}=m_{0}^{c}+m_{0}^{p}$ satisfy properties (i)-(iv) of this theorem with $Y_{0}:=\frac{d m_{0}^{c}}{d \mathbb{P}}$. Let $A$ be one of the measurable sets with probability one given in the definition of cyclic anticomonotonicity. We fix an $\omega_{0} \in A$ and, motivated by the proof of Theorem 24.8 in [28], we consider the function $U$ on $\mathbb{R}_{+}^{d}$ by:

$$
U(x):=\inf \left\{\left\langle x-X_{0}\left(\omega_{p}\right), Y_{0}\left(\omega_{p}\right)\right\rangle+\cdots+\left\langle X_{0}\left(\omega_{1}\right)-X_{0}\left(\omega_{0}\right), Y_{0}\left(\omega_{0}\right)\right\rangle\right\}, \quad x \in \mathbb{R}_{+}^{d}
$$

where the infimum is taken over all finite sets $\left(\omega_{0}, \omega_{1}, \ldots, \omega_{p}\right)$ ( $p$ arbitrary) of elements of $A$. Moreover, we set $U(x)=-\infty$ for $x \notin \mathbb{R}_{+}^{d}$, so that we have in particular that $\operatorname{dom}(U) \subset \mathbb{R}_{+}^{d}$.

We will prove that $U$ belongs to $\mathcal{U}$ and that $X_{0}$ is efficient for $U$ along the following steps. First, we prove in (1) that $U$ is a proper closed concave function. Then for each $\omega \in A$, we note that $Y_{0}(\omega) \in \partial U\left(X_{0}(\omega)\right)$, which corresponds to property (2) below, and deduce in (3) that $U$ is nondecreasing with respect to the preorder induced by $\mathbb{R}_{+}^{d}$. Finally, establishing in (4) that $\overline{\operatorname{dom} U}=\mathbb{R}_{+}^{d}$ and in (5) that $U$ is strictly increasing, we deduce in (6) that $U$ belongs to $\mathcal{U}$ and $X_{0}$ is efficient for $U$.

(1) Since $U$ is an infimum of a collection of affine functions, $U$ is a closed concave function. Moreover, by construction we have $U\left(X_{0}\left(\omega_{0}\right)\right)=0$ by cyclic anticomonotonicity of $X_{0}$ and $Y_{0}$ and hence $U$ is proper.

(2) Let $\omega \in A$, it is enough to show that for any $\alpha>U\left(X_{0}(\omega)\right)$, and any $z \in \mathbb{R}^{d}$, the following property holds:

$$
U(z)<\alpha+\left\langle Y_{0}(\omega), z-X_{0}(\omega)\right\rangle .
$$

By definition of $U$, there exists some $\omega_{i}, i=1, \ldots, p$ such that:

$$
\alpha>\left\langle Y_{0}\left(\omega_{p}\right), X_{0}(\omega)-X_{0}\left(\omega_{p}\right)\right\rangle+\cdots+\left\langle Y_{0}\left(\omega_{0}\right), X_{0}\left(\omega_{1}\right)-X_{0}\left(\omega_{0}\right)\right\rangle .
$$

By definition of $U$ and setting $\omega_{p+1}=\omega$, we deduce:

$$
U(z) \leq\left\langle Y_{0}\left(\omega_{p}\right), z-X_{0}\left(\omega_{p+1}\right)\right\rangle+\cdots+\left\langle Y_{0}\left(\omega_{0}\right), X_{0}\left(\omega_{1}\right)-X_{0}\left(\omega_{0}\right)\right\rangle<\alpha+\left\langle Y_{0}\left(\omega_{p}\right), z-X_{0}(\omega)\right\rangle
$$

and this proves that $Y_{0}(\omega) \in \partial U\left(X_{0}(\omega)\right)$.

(3) We prove that $U\left(x^{\prime}\right) \geq U(x)$ as soon as $x^{\prime} \geq x$, i.e. $x^{\prime}-x \in \mathbb{R}_{+}^{d}$. Indeed, using $U$ 's definition, we can choose $\varepsilon>0$ and $\left(\omega_{0}, \omega_{1}, \ldots, \omega_{p}\right)$ such that

$$
\left\langle x^{\prime}-X_{0}\left(\omega_{p}\right), Y_{0}\left(\omega_{p}\right)\right\rangle+\cdots+\left\langle X_{0}\left(\omega_{1}\right)-X_{0}\left(\omega_{0}\right), Y_{0}\left(\omega_{0}\right)\right\rangle \leq U\left(x^{\prime}\right)+\varepsilon .
$$

Using the definition of $U$ once more, we have

$$
\begin{aligned}
U(x) & \leq\left\langle x-X_{0}\left(\omega_{p}\right), Y_{0}\left(\omega_{p}\right)\right\rangle+\cdots+\left\langle X_{0}\left(\omega_{1}\right)-X_{0}\left(\omega_{0}\right), Y\left(\omega_{0}\right)\right\rangle \\
& \leq U\left(x^{\prime}\right)+\varepsilon+\left\langle x-x^{\prime}, Y_{0}\left(\omega_{p}\right)\right\rangle
\end{aligned}
$$

and since $x^{\prime}-x \in \mathbb{R}_{+}^{d}$ and $Y\left(\omega_{p}\right) \in \operatorname{int} \mathbb{R}_{+}^{d}$, we have $U(x) \leq U\left(x^{\prime}\right)+\varepsilon$. Being $\varepsilon>0$ arbitrary, we can conclude that $U$ is increasing for the preorder induced by $\mathbb{R}_{+}^{d}$.

(4) First, notice that $U$ is finite on each $X_{0}(\omega)$ for $\omega \in A$ 2 Consider first the case where ess inf $\left\|X_{0}\right\|=0$ and take some $x \in \operatorname{int} \mathbb{R}_{+}^{d}$. We can choose a $\omega \in A$ such that $X_{0}(\omega) \leq x$, and deduce that $U\left(X_{0}(\omega)\right) \leq U(x)$, i.e. $x \in \operatorname{dom}(U)$ so implying that $\operatorname{int} \mathbb{R}_{+}^{d} \subset \operatorname{dom} U$. Since $\operatorname{dom} U \subset \mathbb{R}_{+}^{d}$ (by definition of $U$ ), we have that $\overline{\operatorname{dom} U}=\mathbb{R}_{+}^{d}$. If, on the contrary, one has ess inf $\left\|X_{0}\right\|>0$, then, by property (iv) in the statement we have ess sup $\left\|Y_{0}\right\|<\infty$. Assume that for some $x \in \operatorname{int} \mathbb{R}_{+}^{d}, U(x)=-\infty$, and choose a sequence $x_{n} \in \operatorname{dom} U$ such that $u_{n}:=U\left(x_{n}\right)$ goes to $-\infty$ as $n \rightarrow+\infty$. Proceeding as in (2) above, for all $n \geq 1$ there exists some finite sequence $\left(\omega_{i}\right)_{i=1}^{p}$ (depending on the chosen $n$ ) such that

$$
u_{n}>\left\langle Y_{0}\left(\omega_{p}\right), z_{n}-X_{0}\left(\omega_{p}\right)\right\rangle+\cdots+\left\langle Y_{0}\left(\omega_{0}\right), X_{0}\left(\omega_{1}\right)-X_{0}\left(\omega_{0}\right)\right\rangle .
$$

\footnotetext{
${ }^{2}$ Since $Y_{0}(\omega) \in \partial U\left(X_{0}(\omega)\right)$ for all $\omega \in A$, we have $\partial U\left(X_{0}(\omega)\right) \neq \emptyset$. Thus, the fact that $\operatorname{dom}(\partial U) \subset \operatorname{dom}(U)$ implies that $X_{0}(\omega) \in \operatorname{dom}(\partial U) \subset \operatorname{dom}(U)$, so that $U\left(X_{0}(\omega)\right)>-\infty$.
} 
By definition of $U$ ad setting $\omega_{p+1}=\omega_{0}$, we have

$$
\begin{aligned}
U\left(X_{0}\left(\omega_{0}\right)\right) & \leq\left\langle Y_{0}\left(\omega_{p}\right), X_{0}\left(\omega_{0}\right)-X_{0}\left(\omega_{p+1}\right)\right\rangle+\cdots+\left\langle Y_{0}\left(\omega_{0}\right), X_{0}\left(\omega_{1}\right)-X_{0}\left(\omega_{0}\right)\right\rangle \\
& \leq u_{n}+\left\langle Y_{0}\left(\omega_{p}\right), X_{0}\left(\omega_{0}\right)-z_{n}\right\rangle \\
& \leq u_{n}+M\left\|X_{0}\left(\omega_{0}\right)-z_{n}\right\|,
\end{aligned}
$$

where $M \geq \operatorname{ess} \sup \left\|Y_{0}\right\|$. This leads to a contradiction since the right hand side above goes to $-\infty$ as $n \rightarrow+\infty$ while the left hand side stays finite.

(5) Let's take $x$ and $x^{\prime}$ such that $x^{\prime}>x$. First, suppose that $X_{0} \leq x^{\prime}$ almost surely. In particular ess sup $\left\|X_{0}\right\|<\infty$, and from property (iv) we have ess $\inf \left|Y_{0}^{j}\right|>0$ for some $j$. As in (3), we can choose $\varepsilon>0$ and $\omega \in A$ such that :

$$
U(x) \leq U\left(x^{\prime}\right)+\varepsilon+\left\langle x-x^{\prime}, Y(\omega)\right\rangle .
$$

Therefore, $\left\langle x^{\prime}-x, Y_{0}(\omega)\right\rangle \geq\left(x^{\prime j}-x^{j}\right) \operatorname{essinf} Y_{0}^{j}>0$ a.s. so that $U(x)<U\left(x^{\prime}\right)$. Now, suppose that there exists $\omega \in A$ such that $X_{0}(\omega) \not \leq x^{\prime}$. We can nonetheless choose $x^{\prime} \geq x^{\prime \prime} \geq x$ with $x^{\prime \prime}=\left\langle\alpha, X_{0}(\omega)-x^{\prime}\right\rangle$ for a suitable $\alpha \in \mathbb{R}^{d}$. Thus, we have

$$
U\left(x^{\prime \prime}\right) \geq U(x)+\left\langle Y_{0}(\omega), x^{\prime \prime}-x\right\rangle .
$$

But $Y_{0}(\omega) \in \operatorname{int} \mathbb{R}_{+}^{d}$ (property (i) of this theorem) and consequently $\left\langle Y_{0}\left(\omega_{0}\right), x^{\prime \prime}-x\right\rangle>0$. We can conclude that $U\left(x^{\prime}\right) \geq U\left(x^{\prime \prime}\right)>U(x)$.

(6) Let $X \in \mathcal{A}_{T}^{x_{0}}$. Since, $Y_{0} \in \partial U\left(X_{0}\right)$ almost surely, we have:

$$
\mathbb{E}[U(X)]-\mathbb{E}\left[U\left(X_{0}\right)\right] \leq \mathbb{E}\left[Y_{0}\left(X-X_{0}\right)\right] \leq 0 .
$$

Therefore $X_{0}$ is efficient for $U$ and $x_{0}$. This ends the proof.

Remark 4.1 A careful inspection of the proof of the previous theorem reveals that the assumption of upper semicontinuity in the definition of $\mathcal{U}$ can be replaced by the minimal requirement of measurability.

Remark 4.2 In many papers on utility maximization under transaction costs (see, e.g., [2, 6, 20]), the agent liquidates his terminal portfolio to the first asset. As in the two papers [1, 3], one could include the slightly more general case of agents willing to liquidate their final portfolios to the first $k$ assets with $k \leq d$. It suffices to consider utility functions of the form $U\left(x_{1}, \ldots, x_{d}\right)=U_{k}\left(x_{1}, \ldots, x_{k}\right)$. In this case, the characterization can be proved performing the same arguments with respect to the first $k$ coordinates and using the fact that, in this case, an optimal portfolio $X_{0}$ is zero everywhere but in the first $k$ components (which has been proved in [3]). Of course, the property of cyclic anticomonotonicity would hold between the first $k$ coordinates of $X_{0}$ and the first $k$ coordinates of at least one $Y=d m^{c} / d \mathbb{P}$ with $m=m^{c}+m^{p} \in \mathcal{D}$.

\section{$5 \quad$ Utility price and inefficiency size of a contingent claim}

In this section, we will consider contingent claims that might be inefficient, i.e. they are not optimal for any rational investors, and we want to quantify their inefficiency, that is to measure how far are they from being optimal for one agent. We are going to do that in the spirit of Dybvig's papers [9, 10] and Jouini and Kallal [18, where the inefficiency size is the difference between the initial investment needed to get the claim at maturity and the largest amount needed by any rational agent to get a possibly different claim getting the same expected utility level.

To do so, we need some preliminary definitions. Given an arbitrary and not necessarily efficient claim $X_{0} \in \mathcal{A}_{T}^{x_{0}}$ attainable starting from some initial endowment $x_{0}$, we denote by $\pi\left(X_{0}, x_{0}\right)$ the smallest proportion of portfolio $x_{0}$ allowing to super-hedge $X_{0}$, i.e.

$$
\pi\left(X_{0}, x_{0}\right):=\inf \left\{\alpha \in[0,1]: X_{0} \in \mathcal{A}_{T}^{\alpha x_{0}}\right\} .
$$


Let us introduce the set $\mathcal{B}^{U}\left(X_{0}\right)$ of all contingent claims which are better than $X_{0}$ for an agent with the utility function $U$ (the notation $\mathcal{B}$ clearly stands for 'better'), i.e.

$$
\mathcal{B}^{U}\left(X_{0}\right)=\left\{X \in L^{0}\left(\mathbb{R}_{+}^{d}\right): \mathbb{E}[U(X)] \geq \mathbb{E}\left[U\left(X_{0}\right)\right]\right\} .
$$

Notice that if $X \sim X_{0}$ then $\mathcal{B}^{U}(X)=\mathcal{B}^{U}\left(X_{0}\right)$ for all utility functions $U \in \mathcal{U}$.

Definition 5.1 Let $X_{0} \in \mathcal{A}_{T}^{x_{0}}$ be an attainable contingent claim with $x_{0} \in \mathcal{I}$. The utility price of $X_{0}$ with respect to the initial portfolio $x_{0}$, denoted $P^{\mathcal{U}}\left(X_{0}, x_{0}\right)$, is defined as the smallest proportion of $x_{0}$ needed for any agent to fund an admissible strategy in $\mathcal{A}$ giving at least the same expected utility as $X_{0}$, i.e.

$$
P^{\mathcal{U}}\left(X_{0}, x_{0}\right):=\sup _{U \in \mathcal{U}} \inf _{X \in \mathcal{B}^{U}\left(X_{0}\right)} \pi\left(X, x_{0}\right) .
$$

The inefficiency size is defined as $I^{\mathcal{U}}\left(X_{0}, x_{0}\right):=1-P^{\mathcal{U}}\left(X_{0}, x_{0}\right) \in[0,1]$.

The previous definition has the following interpretation: a positive inefficiency size for $X_{0}$, or equivalently a "utility price" strictly less than 1 , means that all rational agents can invest strictly less that $x_{0}$ and still get a final contingent claim giving them the same or even a higher expected utility level. The quantity $I^{\mathcal{U}}\left(X_{0}, x_{0}\right)$ measures how far the claim $X_{0}$ is from being efficient and does it in a "universal" manner since it is independent of agents' preferences. Examples of inefficient portfolios in a market model with a constant non zero bid-ask spread (as well as many other frictions) are provided and discussed in Subsection 2.1 in Jouini and Kallal [18.

Remark 5.1 Notice that if $X_{0}$ is efficient for an initial portfolio $x_{0}$ and some utility function $U \in \mathcal{U}$, we have $\pi\left(X_{0}, x_{0}\right)=1$ for all $X \in \mathcal{B}^{U}\left(X_{0}\right)$, implying that $P^{\mathcal{U}}\left(X_{0}, x_{0}\right)=13^{3}$ This is consistent with the use of $I^{\mathcal{U}}\left(X_{0}, x_{0}\right)=1-P^{\mathcal{U}}\left(X_{0}, x_{0}\right)$ as a measure of the possible inefficiency of a given terminal portfolio $X_{0}$.

Let $\mathcal{D}^{\perp}\left(x_{0}\right)$ denote the subset of $\mathcal{D}$ given by $\mathcal{D}^{\perp}\left(x_{0}\right)=\left\{m \in \mathcal{D}: m(\Omega) \in K_{0}^{*}\left(x_{0}\right)\right\}$, where $K_{0}^{*}\left(x_{0}\right)=$ $\left\{z \in K_{0}^{*}: z=\frac{x_{0}}{\left\|x_{0}\right\|^{2}}+z^{\perp},\left\langle z^{\perp}, x_{0}\right\rangle=0\right\}$ when $x_{0}$ is nonzero, and $K_{0}^{*}(0)=K_{0}^{*}$ otherwise. Notice that $K_{0}^{*}\left(x_{0}\right)$ is a closed convex non empty set and that whenever $x_{0} \in \mathbb{R}_{+}^{d}$ we have $K_{0}^{*}=\operatorname{cone}\left(K_{0}^{*}\left(x_{0}\right)\right)$. Using the duality result in Theorem 3.1 (super-replication theorem), we can evaluate as a first step the smallest proportion of the initial portfolio $x_{0}$ needed to hedge the contingent claim $X_{0}$. The next lemma contains a dual characterization of it. Its proof has been relegated in the Appendix.

Lemma 5.1 The smallest amount of portfolio $x_{0}$ needed to hedge the contingent claim $X_{0}$ is given by

$$
\pi\left(X_{0}, x_{0}\right)=\sup _{m \in \mathcal{D}^{\perp}\left(x_{0}\right)} m\left(X_{0}\right)=\sup _{m \in \mathcal{D}^{\perp}\left(x_{0}\right) \cap \operatorname{ca}\left(\mathbb{R}_{+}^{d}\right)} m\left(X_{0}\right) .
$$

As a consequence, $X_{0} \in \mathcal{A}_{T}^{x_{0}}$ if and only if

$$
\sup _{m \in \mathcal{D}^{\perp}\left(x_{0}\right)} m\left(X_{0}\right) \leq 1
$$

Now, we can state the main result of this section, giving a dual representation of the utility price. In order to do so, it is convenient to introduce the following set

$$
\mathcal{B}\left(X_{0}\right):=\bigcap_{U \in \mathcal{U}} \mathcal{B}^{U}\left(X_{0}\right)=\left\{X \in L^{0}\left(\mathbb{R}_{+}^{d}\right): \mathbb{E}[U(X)] \geq \mathbb{E}\left[U\left(X_{0}\right)\right], \forall U \in \mathcal{U}\right\}
$$

which describes the set of all (positive) contingent claims which are better than $X_{0}$ for all rational agents.

\footnotetext{
${ }^{3}$ Indeed, assume that $\pi\left(X_{0}, x_{0}\right)<1$ for some $X \in \mathcal{B}^{U}\left(X_{0}\right)$. Since $X_{0}$ is efficient for some $x_{0}$ and $U \in \mathcal{U}$, we have that $X_{0}$ is a maximizer for an agent having utility function $U$ and an initial portfolio $x_{0}$. Moreover, by definition of $\pi\left(X_{0}, x_{0}\right)$, the initial portfolio $\pi\left(X_{0}, x_{0}\right) x_{0}<x_{0}$ leads to $X$ as well. In other terms, the initial wealth $x_{0}$ may lead to the terminal portfolio $X_{0}+\left(1-\pi\left(X_{0}, x_{0}\right)\right) x_{0}>X$. Since $U$ is strictly increasing, this contradicts the fact that $X_{0}$ is a maximizer. Thus, when $X_{0}$ is efficient its utility price.
} 
Theorem 5.1 Let $x_{0} \in \mathcal{I}$ be an initial portfolio and let $X_{0}$ be a contingent claim in $\mathcal{A}_{T}^{x_{0}}$. Moreover, assume that the c.d.f. of $X_{0}$ is continuous. Thus, the utility price of $X_{0}$ satisfies the following properties:

$$
\begin{aligned}
P^{\mathcal{U}}\left(X_{0}, x_{0}\right) & =\inf _{X \in \mathcal{B}\left(X_{0}\right)} \pi\left(X, x_{0}\right) \\
& =\sup _{m \in \mathcal{D}^{\perp}\left(x_{0}\right)} \inf _{X \in \mathcal{B}\left(X_{0}\right)} m(X) .
\end{aligned}
$$

Before proving this theorem, let us comment about its content. First of all, the dual representation (5.7) states that the "utility price" can be viewed as the largest proportion of the initial investment $x_{0}$ necessary for any rational agent to get a (possibly different) claim with the same expected utility level, which extends to our setting previous results of Jouini and Kallal [18.

Another consequence of the theorem above is the representation of the utility price as an infimum over the set $\mathcal{B}\left(X_{0}\right)$ of all contingent claim preferred to $X_{0}$ by all rational agents. Notice that $\mathcal{B}\left(X_{0}\right)$ contains the set $\overline{\operatorname{conv}} \mathcal{L}\left(X_{0}\right)+L^{0}\left(\mathbb{R}_{+}^{d}\right)$. The latter set includes random variables that might not have the same law as $X_{0}$. This corresponds to a phenomenon that has already been observed in Theorem 2 of Jouini and Kallal [18] in a less general frictional setting, i.e. there might exist a contingent claim $\tilde{X}_{0}$ giving at least the same expected utility as $X_{0}$ at the same initial cost for all rational agents, which is not necessarily distributed as $X_{0}$, as in the case of complete and frictionless markets (see Dybvig [9]). This implies in particular that for some utility function $U \in \mathcal{U}$, expected utility of $\tilde{X}_{0}$ may be strictly bigger than the expected utility of $X_{0}$ (see the example in the Appendix of [18]).

Now, we can turn to the proof of Theorem 5.1, which is based on the following preliminary lemma, whose technical proof is postponed to the Appendix.

Lemma 5.2 Let $X_{0} \in \mathcal{A}_{T}^{x_{0}}$ with $x_{0} \in \mathcal{I}$. The following two properties hold:

(i) For any countably additive measure $m \in \mathcal{D}^{\perp}\left(x_{0}\right) \cap \mathrm{ca}\left(\mathbb{R}_{+}^{d}\right)$ with $Y:=\frac{d m}{d \mathbb{P}} \in \operatorname{int} \mathbb{R}_{+}^{d}$ a.s., there exists $\tilde{X}_{0} \sim X_{0}$ such that $\tilde{X}_{0}$ and $Y$ are cyclically anticomonotonic and

$$
\sup _{U \in \mathcal{U}} \inf _{X \in \mathcal{B}^{U}\left(X_{0}\right)} \mathbb{E}[Y X]=\min _{X \in \mathcal{B}\left(X_{0}\right)} \mathbb{E}[Y X]=\mathbb{E}\left[Y \tilde{X}_{0}\right]
$$

(ii) We have

$$
\inf _{X \in \mathcal{B}\left(X_{0}\right)} \pi\left(X, x_{0}\right)=\sup _{m \in \mathcal{D}^{\perp}\left(x_{0}\right)} \inf _{X \in \mathcal{B}\left(X_{0}\right)} m(X) .
$$

We conclude this section with the proof of Theorem 5.1 .

Proof of Theorem 5.1. First, observe that the same arguments used to prove (5.9) lead to the same conclusion with $\mathcal{B}^{U}\left(X_{0}\right)$ replacing $\mathcal{B}\left(X_{0}\right)$, yielding

$$
P^{\mathcal{U}}\left(X_{0}, x_{0}\right)=\sup _{U \in \mathcal{U}} \inf _{X \in \mathcal{B}^{U}\left(X_{0}\right)} \pi\left(X, x_{0}\right)=\sup _{U \in \mathcal{U}} \sup _{m \in \mathcal{D}^{\perp}\left(x_{0}\right)} \inf _{X \in \mathcal{B}^{U}\left(X_{0}\right)} m(X) .
$$

Therefore we have

$$
P^{\mathcal{U}}\left(X_{0}, x_{0}\right) \geq \sup _{m \in \mathcal{D}_{+}^{\perp}\left(x_{0}\right)} \sup _{U \in \mathcal{U}} \inf _{X \in \mathcal{B}^{U}\left(X_{0}\right)} m(X)
$$

where $\mathcal{D}_{+}^{\perp}\left(x_{0}\right)=\left\{m \in \mathcal{D}^{\perp}\left(x_{0}\right) \cap \mathrm{ca}\left(\mathbb{R}_{+}^{d}\right): \frac{d m}{d \mathbb{P}} \in \operatorname{int} \mathbb{R}_{+}^{d}\right\}$. From 5.8, we have

$$
\sup _{U \in \mathcal{U}} \inf _{X \in \mathcal{B}^{U}\left(X_{0}\right)} m(X)=\min _{X \in \mathcal{B}\left(X_{0}\right)} m(X)
$$


for $m \in \mathcal{D}_{+}^{\perp}\left(x_{0}\right)$ and therefore

$$
P^{\mathcal{U}}\left(X_{0}, x_{0}\right) \geq \sup _{m \in \mathcal{D}_{+}^{\perp}\left(x_{0}\right)} \inf _{X \in \mathcal{B}\left(X_{0}\right)} m(X) .
$$

Moreover, let $m^{\prime} \in \mathcal{D}_{+}^{\perp}\left(x_{0}\right), m \in \mathcal{D}^{\perp}\left(x_{0}\right) \cap \mathrm{ca}\left(\mathbb{R}_{+}^{d}\right)$, and $m_{\lambda}=\lambda m+(1-\lambda) m^{\prime}$ for $\lambda \in[0,1)$. We have $m_{\lambda} \in \mathcal{D}_{+}^{\perp}\left(x_{0}\right)$ and for all $X \in \mathcal{B}\left(X_{0}\right), \lim _{\lambda \uparrow 1} m_{\lambda}(X)=m(X)$. We deduce that:

$$
\sup _{m \in \mathcal{D}^{\perp}\left(x_{0}\right)} \inf _{X \in \mathcal{B}\left(X_{0}\right)} m(X)=\sup _{m \in \mathcal{D}_{+}^{\perp}\left(x_{0}\right)} \inf _{X \in \mathcal{B}\left(X_{0}\right)} m(X) .
$$

Now, we can use equality $(5.9)$ to obtain

$$
P^{\mathcal{U}}\left(X_{0}, x_{0}\right) \geq \inf _{X \in \mathcal{B}\left(X_{0}\right)} \sup _{m \in \mathcal{D}^{\perp}\left(x_{0}\right)} m(X) .
$$

The reverse inequality is straightforward and we conclude that

$$
P^{\mathcal{U}}\left(X_{0}, x_{0}\right)=\inf _{X \in \mathcal{B}\left(X_{0}\right)} \sup _{m \in \mathcal{D}^{\perp}\left(x_{0}\right)} m(X)=\inf _{X \in \mathcal{B}\left(X_{0}\right)} \pi\left(X, x_{0}\right)
$$

i.e. we have just proved property (5.6). Finally, the min-max property $(5.9)$ yields

$$
P^{\mathcal{U}}\left(X_{0}, x_{0}\right)=\sup _{m \in \mathcal{D}^{\perp}\left(x_{0}\right)} \inf _{X \in \mathcal{B}\left(X_{0}\right)} m(X)
$$

which ends the proof of the theorem.

\section{Conclusion}

In this paper we extend Dybvig's characterization of efficient portfolios to a very general continuous-time multi-currency market model with proportional transaction costs as those portfolios that are (cyclically) anticomonotonic with respect to some consistent price system. Moreover, we study the notion of utility price and the related one of inefficiency size of a given claim and gives a dual characterization of the "utility price" as the largest proportion of the market price necessary for all agents to reach the same expected utility level. The study of efficient portfolios in more concrete market models (e.g. Black-Scholes model with constant proportional transaction costs) and the use of our characterization to investigate the inefficiency of certain claims is interesting on its own and it does not reduce to a simple application of the abstract results of this paper. It is then postponed to future research.

\section{A Appendix}

This appendix collects the proofs of some technical results that have been used throughout the paper.

Proof of Proposition 4.1. It is straightforward that cyclic anticomonotonicity implies 4.3. Conversely, assume that $X$ and $Y$ satisfy property (4.3). To obtain that $X$ and $Y$ are cyclically anticomonotonic, it suffices to prove that there exist two random vectors $X^{\prime}, Y^{\prime}$ with $X^{\prime}=X$ and $Y^{\prime}=Y$ a.s., such that $X^{\prime}$ and $Y^{\prime}$ are cyclically anticomonotonic. Denote $X=\left(X^{1}, \ldots, X^{d}\right)$ and $Y=\left(Y^{1}, \ldots, Y^{d}\right)$, and consider the product set for $n \in \mathbb{N}^{*}$ :

$$
\mathbb{N}_{n 2^{n}}^{d}:=\left\{1, \ldots, n 2^{n}\right\} \times \ldots \times\left\{1, \ldots, n 2^{n}\right\} .
$$

Then, for each vector of integers $I=\left(I^{1}, \ldots, I^{d}\right)$ and $K=\left(K^{1}, \ldots, K^{d}\right)$ in $\mathbb{N}_{n 2^{n}}^{d}$, define the sets $A_{I}$ and $B_{K}$ by

$$
A_{I}:=\bigcap_{1 \leq i \leq d}\left\{\frac{I^{i}}{2^{n}} \leq X^{i}<\frac{I^{i}+1}{2^{n}}\right\}, \quad B_{K}:=\bigcap_{1 \leq j \leq d}\left\{\frac{I^{j}}{2^{n}} \leq Y^{j}<\frac{I^{j}+1}{2^{n}}\right\} .
$$


in such a way that the family $\left\{A_{I} \cap B_{K}:(I, K) \in\left(N_{n 2^{n}}^{d}\right)^{2}\right\}$ is a partition of the set $\left\{(X, Y) \in[0, n]^{d} \times[0, n]^{d}\right\}$. The rest of the proof is structured in three main steps.

Step 1: construction of $X^{\prime}$. We define

$$
X_{n}^{i}:=\sum_{(I, K) \in P_{n}} \frac{I^{i}}{2^{n}} \mathbf{1}_{A_{I}} \cap B_{K}
$$

with $P_{n}:=\left\{(I, K) \in\left(\mathbb{N}_{n 2^{n}}^{d}\right)^{2}: \mathbb{P}\left(A_{I} \cap B_{K}\right)>0\right\}$. Defining

$$
N_{n}:=\left(\bigcap_{(I, K) \in P_{n}}\left(A_{I} \cap B_{K}\right)\right)^{C}
$$

one can see that when $\omega \notin N_{n}$, we have $\left\|X^{n}(\omega)-X(\omega)\right\| \leq 2^{-n}$ for every $n \in \mathbb{N}$. Thus, for every $\omega \in \Omega$, $X^{n}$ converges and we can define its limit by setting

$$
X^{\prime}(\omega):=\lim _{n \rightarrow+\infty} X_{n}(\omega) .
$$

By construction, $X^{\prime}=X$ a.s., since $\mathbb{P}\left(N_{n}\right) \rightarrow 0$. Indeed, one has $\mathbb{P}\left(N_{n}\right)=\mathbb{P}(X \geq n \mathbf{1}, Y \geq n \mathbf{1})$ which goes to 0 as $n \rightarrow+\infty$, being both $X, Y$ finite valued.

Step 2: construction of $Y^{\prime}$. In the same way, we define

$$
Y_{n}^{j}=\sum_{(I, K) \in P_{n}} \frac{K^{j}}{2^{n}} \mathbf{1}_{A_{I} \cap B_{K}} .
$$

If $\omega \notin N_{n}$, we have $\left\|Y^{n}(\omega)-Y(\omega)\right\| \leq 2^{-n}$ for every $n \in \mathbb{N}$. Therefore, for every $\omega, Y^{n}$ converges and we can define its limit setting

$$
Y^{\prime}(\omega):=\lim _{n \rightarrow+\infty} Y_{n}(\omega)
$$

yielding that $Y^{\prime}=Y$ almost surely.

Step 3: conclusion. The two sequences $Y_{n}$ and $X_{n}$ have been constructed in order to have cyclically anticomonotonic random vectors in the limit. Indeed, let $\Omega_{n}=\Omega \backslash N_{n}$ and $p \in \mathbb{N}^{*}$. For $\left(\omega_{1}, \omega_{2}\right) \in \Omega_{n}^{2}$, we have

$$
\begin{aligned}
\left\langle Y^{n}\left(\omega_{1}\right), X^{n}\left(\omega_{1}\right)-X^{n}\left(\omega_{2}\right)\right\rangle-\left\langle Y\left(\omega_{1}\right), X\left(\omega_{1}\right)-X\left(\omega_{2}\right)\right\rangle= & \left\langle Y^{n}\left(\omega_{1}\right)-Y\left(\omega_{1}\right), X^{n}\left(\omega_{1}\right)-X^{n}\left(\omega_{2}\right)\right\rangle \\
& +\left\langle Y\left(\omega_{1}\right), X^{n}\left(\omega_{1}\right)-X\left(\omega_{1}\right)\right\rangle \\
& -\left\langle Y\left(\omega_{1}\right), X^{n}\left(\omega_{2}\right)-X\left(\omega_{2}\right)\right\rangle \\
\leq & \frac{n}{2^{n}}+\frac{n}{2^{n}}+\frac{n}{2^{n}} .
\end{aligned}
$$

Thus, for every $\left(\omega_{1}, \ldots, \omega_{p+1}\right) \in \Omega_{n}^{p+1}$ with $\omega_{p+1}=\omega_{1}$, we have

$$
\sum_{i=1}^{p}\left\langle Y^{n}\left(\omega_{i}\right), X^{n}\left(\omega_{i}\right)-X^{n}\left(\omega_{i+1}\right)\right\rangle-\sum_{i=1}^{p}\left\langle Y\left(\omega_{i}\right), X\left(\omega_{i}\right)-X\left(\omega_{i+1}\right)\right\rangle \leq 3 p \frac{n}{2^{n}} .
$$

Consider now the function

$$
g_{n}\left(\omega_{1}, \ldots, \omega_{p}\right):=\left\langle Y_{n}\left(\omega_{1}\right), X_{n}\left(\omega_{1}\right)-X_{n}\left(\omega_{2}\right)\right\rangle+\cdots+\left\langle Y_{n}\left(\omega_{p}\right), X_{n}\left(\omega_{p}\right)-X_{n}\left(\omega_{1}\right)\right\rangle .
$$


It is constant on the set $\times_{i=1}^{p} A_{I^{i}} \cap B_{K^{i}}$, for $\left(I^{i}, K^{i}\right) \in P_{n}, i=1, \ldots, p$. Thus, we necessarily have

$$
g_{n}\left(\omega_{1}, \ldots, \omega_{p}\right) \leq 3 p \frac{n}{2^{n}} .
$$

Indeed, if it was not the case, equation A.1 would yield that for some $\left(I^{i}, K^{i}\right) \in P_{n}, i=1, \ldots, p$, and for all $\left(\omega_{1}, \ldots, \omega_{p}\right) \in \times_{i=1}^{p} A_{I^{i}} \cap B_{K^{i}}$ - a strictly positive probability set, by construction - the following inequality holds:

$$
\left\langle Y\left(\omega_{1}\right), X\left(\omega_{1}\right)-X\left(\omega_{2}\right)\right\rangle+\cdots+\left\langle Y\left(\omega_{p}\right), X\left(\omega_{p}\right)-X\left(\omega_{1}\right)\right\rangle>0
$$

which contradicts equation 4.3 . We conclude that for every $\left(\omega_{1}, \ldots, \omega_{p}\right) \in A^{p}$, where $A:=\Omega \backslash \cap_{n \geq 1} N_{n}$, we have

$$
\left\langle Y^{\prime}\left(\omega_{1}\right), X^{\prime}\left(\omega_{1}\right)-X^{\prime}\left(\omega_{2}\right)\right\rangle+\cdots+\left\langle Y^{\prime}\left(\omega_{p}\right), X^{\prime}\left(\omega_{p}\right)-X^{\prime}\left(\omega_{1}\right)\right\rangle \leq 0
$$

with $\mathbb{P}(A)=1-\lim _{n} \mathbb{P}\left(N_{n}\right)=1$.

Proof of Proposition 4.2. First, distinguish between two cases: If for all $X \in \mathcal{L}\left(X_{0}\right)$ (implying in particular that $X \in L^{0}\left(\mathbb{R}_{+}^{d}\right)$ ) we have $\mathbb{E}[X Y]=+\infty$, there is nothing to prove. Let us turn to the second case when there exists at least one $X \in \mathcal{L}\left(X_{0}\right)$ such that $\mathbb{E}[X Y]<\infty$, so that the infimum in 4.5 is finite.

In the following, we denote $C_{X_{0}}$ a copula of $X_{0}$, and $C_{Y}$ a copula of $Y$ (we refer to Nelson's book 24] for details on copulas). Consider now the set $\mathcal{C}\left(X_{0}, Y\right)$ of all copulas on $\mathbb{R}^{2 d}$, such that for every $C \in \mathcal{C}\left(X_{0}, Y\right)$, the marginal copula of the $d$ first variables is $C_{X_{0}}$, and the marginal copula of the $d$ last variables is $C_{Y}$, i.e.

$$
\begin{aligned}
C\left(u_{1}, u_{2}, \ldots, u_{d}, 1, \ldots, 1\right) & =C_{X}\left(u_{1}, u_{2}, \ldots, u_{d}\right), \\
C\left(1, \ldots, 1, v_{1}, v_{2}, \ldots, v_{d}\right) & =C_{Y}\left(u_{1}, u_{2}, \ldots, u_{d}\right) .
\end{aligned}
$$

It is straightforward to see that the set $\mathcal{C}\left(X_{0}, Y\right)$ is closed with respect to the topology of pointwise convergence on $\mathcal{C}$, the set of all possible copulas on $\mathbb{R}^{2 d}$. Furthermore the set $\mathcal{C}$ is compact with respect to this topology (see Deheuvels [7, Theorem 2.3). Thus the set $\mathcal{C}\left(X_{0}, Y\right)$ is itself compact with respect to the topology of pointwise convergence. Let $X_{n}$ be a sequence in $\mathcal{L}\left(X_{0}\right)$ such that

$$
\lim _{n \rightarrow+\infty} \mathbb{E}\left(Y X_{n}\right)=\inf \left\{\mathbb{E}(Y X): X \in \mathcal{L}\left(X_{0}\right)\right\}
$$

and let $C_{n}$ denote the copula of $\left(X_{n}, Y\right)$. Since $\mathcal{C}\left(X_{0}, Y\right)$ is compact, we can assume without loss of generality. (up to extracting subsequences) that the sequence $C_{n} \in \mathcal{C}\left(X_{0}, Y\right)$ converges pointwise to a copula $C \in$ $\mathcal{C}\left(X_{0}, Y\right)$. Consider the random vector $\tilde{X}_{0}$ such that the copula of $\left(\tilde{X}_{0}, Y\right)$ is $C$. In particular, $\tilde{X}_{0} \in \mathcal{L}\left(X_{0}\right)$. Notice that

$$
\begin{aligned}
\mathbb{E}\left[\left\langle Y, X_{n}\right\rangle\right] & =\sum_{i=1}^{d} \mathbb{E}\left[X_{n}^{i} Y^{i}\right]=\sum_{i=1}^{d} \int_{0}^{\infty} \int_{0}^{\infty} \mathbb{P}\left(X_{n}^{i}>t, Y^{i}>u\right) d t d u \\
& =\sum_{i=1}^{d} \int_{0}^{\infty} \int_{0}^{\infty}\left[1-\mathbb{P}\left(X_{0}^{i} \leq t\right)-\mathbb{P}\left(Y^{i} \leq u\right)-C_{n}^{i}\left(\mathbb{P}\left(X_{0}^{i} \leq t\right), \mathbb{P}\left(Y^{i} \leq u\right)\right)\right] d t d u .
\end{aligned}
$$

where $C_{n}^{i}$ denotes the marginal copula of the vector $\left(X_{n}^{i}, Y^{i}\right)$ and where we used the fact that $X_{n}^{i}$ has the same law as $X_{0}^{i}$ for all $i$. By the pointwise convergence of $C_{n}$ to the copula $C$ of $\left(\tilde{X}_{0}, Y\right)$, we deduce that, for every $u \geq 0, t \geq 0$ and $i \in\{1, \ldots, d\}$, we have

$$
\lim _{n \rightarrow+\infty} C_{n}^{i}\left(\mathbb{P}\left(X_{0}^{i} \leq t\right), \mathbb{P}\left(Y^{i} \leq u\right)\right)=C^{i}\left(\mathbb{P}\left(X_{0}^{i} \leq t\right), \mathbb{P}\left(Y^{i} \leq u\right)\right)
$$

with $C^{i}$ is the marginal copula of the vector $\left(\tilde{X}_{0}^{i}, Y^{i}\right)$. Therefore by Fatou's lemma and the equation A.2, we have

$$
\begin{aligned}
\mathbb{E}\left[\left\langle\tilde{X}_{0}, Y\right\rangle\right] & =\sum_{i=1}^{d} \int_{0}^{\infty} \int_{0}^{\infty} 1-\mathbb{P}\left(X_{0}^{i} \leq t\right)-\mathbb{P}\left(Y^{i} \leq u\right)-C^{i}\left(\mathbb{P}\left(X_{0}^{i} \leq t\right), \mathbb{P}\left(Y^{i} \leq u\right)\right) d t d u \\
& \leq \liminf _{n \rightarrow+\infty} \mathbb{E}\left[\left\langle X_{n}, Y\right\rangle\right] \leq \min \left\{\mathbb{E}[X Y]: X \in \mathcal{L}\left(X_{0}\right)\right\}
\end{aligned}
$$


and thus, $\mathbb{E}\left[\left\langle\tilde{X}_{0}, Y\right\rangle\right]=\min \left\{\mathbb{E}[Y X]: X \in \mathcal{L}\left(X_{0}\right)\right\}$.

Now, let us prove that $\tilde{X}_{0}$ and $Y$ are cyclically anticomonotonic. Suppose that this is not the case. Thus, by Corollary 4.1 there exist $\varepsilon>0, p \geq 1$ and some non negligible measurable sets $\Omega_{1}, \ldots, \Omega_{p}$ such that for all $\left(\omega_{1}, \ldots, \omega_{p}\right) \in \Omega_{1} \times \cdots \times \Omega_{p}$, we have

$$
\left\langle\tilde{X}_{0}\left(\omega_{1}\right)-\tilde{X}_{0}\left(\omega_{2}\right), Y\left(\omega_{1}\right)\right\rangle+\cdots+\left\langle\tilde{X}_{0}\left(\omega_{p}\right)-\tilde{X}_{0}\left(\omega_{1}\right), Y\left(\omega_{p}\right)\right\rangle \geq \varepsilon
$$

and, being the space $(\Omega, \mathcal{F}, \mathbb{P})$ atomless, we can choose the sets $\Omega_{1}, \ldots, \Omega_{p}$ in such a way that $\mathbb{P}\left(\Omega_{1}\right)=$ $\mathbb{P}\left(\Omega_{2}\right)=\cdots=\mathbb{P}\left(\Omega_{p}\right)$. Consider a random vector $X^{\prime}$, distributed as $\tilde{X}_{0}$ with

$$
\left\{\begin{array}{l}
X_{\mid \Omega \backslash \cup_{i=1}^{p} \Omega_{i}}^{\prime}=\left(\tilde{X}_{0}\right)_{\mid \Omega \backslash \cup_{i=1}^{p} \Omega_{i}} \\
X_{\mid \Omega_{i}}^{\prime} \sim\left(\tilde{X}_{0}\right)_{\mid \Omega_{i+1}} \text { for } 1 \leq i \leq p
\end{array}\right.
$$

with the convention $\Omega_{p+1}=\Omega_{1} 4^{4}$ A consequence of such a construction is that $X^{\prime}\left(\Omega_{i}\right)=\tilde{X}_{0}\left(\Omega_{i+1}\right)$ a.s. for all $i=1, \ldots, p$. Since $X^{\prime}$ and $\tilde{X}^{0}$ coincide on $\Omega \backslash \cup_{i=1}^{p} \Omega_{i}$ we have

$$
\mathbb{E}\left[\left\langle X^{\prime}, Y\right\rangle\right]-\mathbb{E}\left[\left\langle\tilde{X}_{0}, Y\right\rangle\right]=\sum_{i=1}^{p} \mathbb{E}\left[\left\langle Y,\left(X^{\prime}-\tilde{X}_{0}\right)_{\mid \Omega_{i}}\right\rangle\right] .
$$

Moreover, we have by construction that $\sum_{i=1}^{p}\left\langle Y,\left(X^{\prime}-\tilde{X}_{0}\right)_{\mid \Omega_{i}}\right\rangle \leq-\varepsilon$, which implies

$$
\mathbb{E}\left[\left\langle X^{\prime}, Y\right\rangle\right]-\mathbb{E}\left[\left\langle\tilde{X}_{0}, Y\right\rangle\right] \leq-p \varepsilon
$$

As a consequence, we have $\mathbb{E}\left[X^{\prime} Y\right]<\mathbb{E}\left[\tilde{X}_{0} Y\right]$, so that $\tilde{X}_{0}$ cannot be the minimizer. This is clearly a contradiction and ends the proof.

Proof of Lemma 5.1. Let $\lambda>0$ be a certain proportion of portfolio $x_{0}$. Using Theorem (3.1), we have that $X_{0}$ can be hedged by the initial holdings vector $\lambda x_{0}$, if and only if:

$$
m\left(X_{0}\right) \leq \lambda\left\langle x_{0}, m(\Omega)\right\rangle, \quad \forall m \in \mathcal{D} .
$$

We claim that the latter condition is equivalent to the following

$$
m\left(X_{0}\right) \leq \lambda\left\langle x_{0}, m(\Omega)\right\rangle, \quad \forall m \in \mathcal{D}^{\perp}\left(x_{0}\right) .
$$

Clearly (A.3) implies A.4. Assume (A.4) and let $m$ be any measure in $\mathcal{D}$. Since $m(\Omega) \in K_{0}^{*}$ and $K_{0}^{*}=$ $\operatorname{cone}\left(K_{0}^{*}\left(x_{0}\right)\right)$, we have $m(\Omega)=\beta z_{0}$ where $\beta \geq 0$ and $z_{0} \in K_{0}^{*}\left(x_{0}\right)$. If $\beta=0$ there is nothing to prove. Consider the case $\beta>0$ and define the measure $m_{\beta}:=m / \beta \in \mathcal{D}$. Moreover, $m_{\beta} \in \mathcal{D}^{\perp}\left(x_{0}\right)$ by construction. The claim is proved. To conclude the proof, notice that whenever $m \in \mathcal{D}^{\perp}\left(x_{0}\right)$ one has $m(\Omega)=x_{0} /\left\|x_{0}\right\|^{2}+z^{\perp}$ with $\left\langle z^{\perp}, x_{0}\right\rangle=0$, so that $\left\langle x_{0}, m(\Omega)\right\rangle=1$. Thus, $X_{0}$ can be hedged by the initial portfolio $\lambda x_{0}$ if and only if

$$
\sup _{m \in \mathcal{D}^{\perp}\left(x_{0}\right)} m\left(X_{0}\right) \leq \lambda
$$

which proves the first equality in 5.3 For the second one, it suffices to use the same arguments using the formulation of Theorem 3.1 in terms of consistent price systems together with the Remark 3.3 in [1] stating a one-to-one correspondence between consistent price systems and elements of $\mathcal{D} \cap \mathrm{ca}\left(\mathbb{R}_{+}^{d}\right)$.

\footnotetext{
${ }^{4}$ A construction of such a random vector $X^{\prime}$ goes as follows: take a random variable $U$ with uniform distribution on $(0,1)$ and set $X^{\prime}=\tilde{X}_{0}$ on $\Omega_{0}$ and, for $i=1, \ldots, n, X_{\mid \Omega_{i}}^{\prime}=F_{i+1}^{-1}\left(U_{i}\right)$ where $U_{i}$ is the restriction of $U$ on $\Omega_{i}$ and $F_{i+1}$ is the c.d.f. of the restriction of $\tilde{X}_{0}$ on $\Omega_{i+1}$. It is easy to verify that $X^{\prime}$ satisfies the properties listed above. Notice that to perform such a construction we need the assumption that $X_{0}$ has a continuous c.d.f..
} 
Proof of Lemma 5.2 (i) Let $Y=\frac{d m}{d \mathbb{P}}$ for some $m \in \mathcal{D}^{\perp}\left(x_{0}\right) \cap$ ca $\left(\mathbb{R}^{d}\right)$. First note that we obviously have $\mathcal{B}\left(X_{0}\right) \subset \mathcal{B}^{U}\left(X_{0}\right)$ for all $U \in \mathcal{U}$, so that

$$
\sup _{U \in \mathcal{U}} \inf _{X \in \mathcal{B}^{U}\left(X_{0}\right)} \mathbb{E}[X Y] \leq \inf _{X \in \mathcal{B}\left(X_{0}\right)} \mathbb{E}[X Y]
$$

We are now going to prove the converse inequality and that the infimum in the right hand side above is attained. By Proposition 4.2, we can choose $\tilde{X}_{0} \sim X_{0}$ such that $\tilde{X}_{0}$ and $Y$ are cyclically anticomonotonic and $\tilde{X}_{0}$ satisfies

$$
\mathbb{E}\left[\tilde{X}_{0} Y\right]=\min \left\{\mathbb{E}[Y X]: X \in \mathcal{L}\left(X_{0}\right)\right\}=: \lambda_{0},
$$

so that the infimum in 5.8 is attained. Notice that $\lambda_{0} \leq 1$ since $m \in \mathcal{D}^{\perp}\left(x_{0}\right)$ and $X_{0} \in \mathcal{A}_{T}^{x_{0}}$, so that $m\left(X_{0}\right)=\mathbb{E}\left[Y X_{0}\right] \leq 1$. At this point, we would be tempted to follow the second part of the proof of Theorem 4.1 to construct a utility function $U \in \mathcal{U}$ such that $\tilde{X}_{0}$ solves

$$
\sup \left\{\mathbb{E}[U(X)]: X \in \mathcal{A}_{T}^{x_{0}}, \mathbb{E}[X Y] \leq \lambda_{0}\right\}
$$

However, it may happen that $\tilde{X}_{0}$ and $Y$ do not satisfy the condition (iv) of Theorem 4.1, i.e.

$$
\operatorname{ess} \sup \|Y\|=\infty \Rightarrow \operatorname{essinf}\left\|\tilde{X}_{0}\right\|=0, \quad \operatorname{ess} \inf \|Y\|=0 \Rightarrow \operatorname{ess} \sup \left\|\tilde{X}_{0}\right\|=\infty .
$$

Nevertheless, for any $\varepsilon>0$ and for all $i=1, \ldots, d$, we can exhibit a random vector $\tilde{X}_{\varepsilon}$ satisfying the following properties

1. $\tilde{X}_{\varepsilon}$ and $Y$ are cyclically anticomonotonic and satisfy the properties A.5 above;

2. $\mathbb{E}\left[Y \tilde{X}_{0}\right]-\varepsilon_{0} \leq \mathbb{E}[Y \tilde{X} \varepsilon] \leq \mathbb{E}\left[Y \tilde{X}_{0}\right]$, for some $0<\varepsilon_{0} \leq \varepsilon$. define

In order to obtain such random variables, one may proceed as follows: for any $\varepsilon>0$ and any $y \in \mathbb{R}_{+}^{d}$,

$$
\tilde{X}_{\varepsilon, y}^{i}:=e^{-\varepsilon Y^{i}} \tilde{X}_{0}^{i} \mathbf{1}_{\{\|Y\|<y\}}+\left(\tilde{X}_{0}+\frac{\varepsilon}{Y^{i}}\right) \mathbf{1}_{\{\|Y\| \geq y\}}, \quad i=1, \ldots, d .
$$

Consider now the function $y \mapsto \psi(y):=\mathbb{E}\left[Y\left(\tilde{X}_{\varepsilon, y}-\tilde{X}_{0}\right)\right]$ and notice that $\psi(y) \rightarrow \varepsilon>0$ as $y \downarrow 0$, while $\psi(y)$ tends to a strictly negative value as $y \rightarrow+\infty$. Thus, one can choose $y=y\left(\varepsilon_{0}\right)$ such that property (b) above is satisfied. It is then easy to check that, by construction, property (a) above is fulfilled as well. Let us denote $\tilde{X}_{\varepsilon}:=\tilde{X}_{\varepsilon, y\left(\varepsilon_{0}\right)}$.

Thus, even though $\tilde{X}_{\varepsilon}$ might not be attainable, we can nonetheless reproduce step-by-step the second part of the proof of Theorem 4.1 , and find a utility function $U_{\varepsilon} \in \mathcal{U}$ such that $\tilde{X}_{\varepsilon}$ solves

$$
\sup \left\{\mathbb{E}\left[U_{\varepsilon}(X)\right]: X \in \mathcal{A}_{T}^{x_{0}}, \mathbb{E}[X Y] \leq \mathbb{E}\left[\tilde{X}_{\varepsilon} Y\right]\right\} .
$$

We first deduce that

$$
\sup _{U \in \mathcal{U}} \inf _{X \in \mathcal{B}^{U}\left(\tilde{X}_{\varepsilon}\right)} \mathbb{E}[X Y] \geq \inf _{X \in \mathcal{B}^{U}\left(\tilde{X}_{\varepsilon}\right)} \mathbb{E}[X Y] \geq \mathbb{E}\left[Y \tilde{X}_{\varepsilon}\right]
$$

Moreover, $\tilde{X}_{0}$ is a contingent claim preferred by each agent to $\tilde{X}_{\varepsilon}$, i.e. $\mathbb{E}\left[U\left(\tilde{X}_{0}\right)\right] \geq \mathbb{E}\left[U\left(\tilde{X}_{\varepsilon}\right)\right]$ for all $U \in \mathcal{U}$. Indeed, let $U \in \mathcal{U}$. Since $Y \in \partial U\left(\tilde{X}_{0}\right)$ (recall that $Y$ and $\tilde{X}_{0}$ are cyclically anticomonotonic), we have

$$
\mathbb{E}\left[U\left(\tilde{X}_{\varepsilon}\right)\right]-\mathbb{E}\left[U\left(\tilde{X}_{0}\right)\right] \leq \mathbb{E}\left[\left\langle Y, \tilde{X}_{\varepsilon}-\tilde{X}_{0}\right\rangle\right] \leq 0
$$

by the right hand side in property (b) above. Therefore $\mathcal{B}^{U}\left(\tilde{X}_{0}\right) \subset \mathcal{B}^{U}\left(\tilde{X}_{\varepsilon}\right)$ for all $U \in \mathcal{U}$, yielding

$$
\sup _{U \in \mathcal{U}} \inf _{X \in \mathcal{B}^{U}\left(\tilde{X}_{0}\right)} \mathbb{E}[X Y] \geq \sup _{U \in \mathcal{U}} \inf _{X \in \mathcal{B}^{U}\left(\tilde{X}_{\varepsilon}\right)} \mathbb{E}[X Y] \geq \mathbb{E}\left[Y \tilde{X}_{\varepsilon}\right] \geq \mathbb{E}\left[Y \tilde{X}_{0}\right]-\varepsilon_{0}
$$


where the second inequality is due to A.6 while the last one comes from the left hand side in property (b) above. Since $\mathcal{B}^{U}\left(\tilde{X}_{0}\right)=\mathcal{B}^{U}\left(X_{0}\right)$, for all $U \in \mathcal{U}$, we finally obtain

$$
\sup _{U \in \mathcal{U}} \inf _{X \in \mathcal{B}^{U}\left(X_{0}\right)} \mathbb{E}[X Y]=\sup _{U \in \mathcal{U}} \inf _{X \in \mathcal{B}^{U}\left(\tilde{X}_{0}\right)} \mathbb{E}[X Y] \geq \min _{X \in \mathcal{B}\left(X_{0}\right)} \mathbb{E}[Y X]-\varepsilon_{0} .
$$

Letting $\varepsilon$ (and so $\varepsilon_{0}$ ) tend to zero ends the proof.

(ii) To prove equality (5.9), it suffices to apply Theorem 3.2 in Pratelli [25], which is a version of min-max theorem well suited to our case since it does not use any compactness assumption. Indeed, $m \mapsto m(X)$ is weak* continuous on the convex set $\mathcal{D}^{\perp}\left(x_{0}\right)$ and $X \mapsto m(X)$ is a linear and so convex function. Moreover $\mathcal{B}\left(X_{0}\right)$ is a nonempty convex subset of $L^{0}\left(\mathbb{R}_{+}^{d}\right)$ and the its level sets $\left\{X \in \mathcal{B}\left(X_{0}\right): m(X) \leq b\right\}$ are closed and bounded with respect to the convergence in probability for any $b \geq 0$ and $m \in \mathcal{D}^{\perp}\left(x_{0}\right) \cap \mathrm{ca}\left(\mathbb{R}_{+}^{d}\right)$. More precisely, Pratelli's theorem is formulated for subsets of $L_{+}^{0}=L^{0}\left(\mathbb{R}_{+}\right)$, the set of all positive real valued random variables. Nonetheless, a careful inspection of his proof reveals that the result still holds in the multidimensional case, i.e. for $L^{0}\left(\mathbb{R}_{+}^{d}\right)$.

\section{References}

[1] G. Benedetti and L. Campi, Multivariate utility maximization with proportional transaction costs and random endowment. SIAM J. Control and Optimization, 50 (2012), 1283-1308.

[2] B. Bouchard, Utility Maximization on the Real Line under Proportional Transaction Costs, Finance Stochast., 6 (2002), 495-516.

[3] L. Campi and M. P. Owen, Multivariate utility maximization with proportional transaction costs. Finance Stochast., 15 (2010), 461-499.

[4] L. Campi and W. Schachermayer, A super-replication theorem in Kabanov's model of transaction costs. Finance Stochast., 10 (2006), 579-596.

[5] G. Carlier, R.-A. Dana and A. Galichon, Pareto efficiency for the concave order and multivariate comonotonicity. Journal of Economic Theory 147 (2012), 207-229.

[6] G. Deelstra, H. Pham and N. Touzi, Dual formulation of the utility maximization problem under transaction costs. Ann. Appl. Probab. 11 (2001), 1353-1383.

[7] P. Deheuvels, Caractérisation complète des lois extrèmes multivariées et de la convergence des types extrèmes. Pub. Inst. Stat. Univ. Paris 23, 3-4 (1978), 1-36.

[8] J. Diestel and J. J. Uhl, Vector measures, Mathematical surveys, no. 15, American Mathematical Society, Providence, Rhode Island, 1977.

[9] P.H. Dybvig, Distributional analysis of portfolio choice. Journal of Business 63, 3 (1988), 369-393.

[10] P.H. Dybvig, Inefficient dynamic portfolio strategies or how to throw away a million dollars in the stock markets. Review of Financial Studies 1, 1 (1988), 67-88.

[11] I. Ekeland, A. Galichon and M. Henry, Comonotonic measures of multivariate risks. Mathematical Finance, 22 (2012), 109-132

[12] I. Ekeland and W. Schachermayer, Law invariant risk measures on $L^{\infty}\left(\mathbb{R}^{d}\right)$. Statistics and Decisions, 28 (2011), 195-225.

[13] P. Guasoni, E. Lépinette and M. Rásonyi, The fundamental theorem of asset pricing under transaction costs. Finance Stochast. 16, 4 (2012), 741-777. 
[14] P. Guasoni, M. Rásonyi and W. Schachermayer, The fundamental theorem of asset pricing for continuous processes under small transaction costs. Annals of Finance 6, 2 (2010), 157-191.

[15] P. Hall and C. C. Heyde. Martingale limit theory and its application. Probability and Mathematical Statistics. Academic Press, Inc. [Harcourt Brace Jovanovich, Publishers], New York-London, 1980.

[16] G. H. Hardy, J. E. Littlewood, and G. Pólya. Inequalities, 2nd Ed., Cambridge University Press, 1952.

[17] E. Jouini and H. Kallal, Martingales and arbitrage in securities markets with transaction costs. J. Econ. Theory, 66 (1995), 178-197.

[18] E. Jouini H. and H. Kallal, Efficient trading strategies in the presence of market frictions. The review of Financial Studies 14, 2 (2001), 343-369.

[19] E. Jouini and V. Portes, Efficient trading strategies. Preprint, 2005. Available at SSRN: http://ssrn.com/abstract $=1000208$

[20] Yu. M. Kabanov, Hedging and liquidation under transaction costs in currency markets, Finance Stochast. 3 (1999), 237-248.

[21] Yu. M. Kabanov and M. Safarian, Markets with Transaction Costs: Mathematical Theory, Springer, Berlin Heidelberg, 2009.

[22] D. Kramkov and W. Schachermayer, The asymptotic elasticity of utility functions and optimal investment in incomplete markets, Ann. Appl. Probab. 9 (1999), 904-950.

[23] D. G. Luenberger, Optimization by vector space methods, Wiley, New York, 1969.

[24] R.B. Nelson. An Introduction to Copulas (Lecture Notes in Statistics No. 139). Springer, New York, 1999.

[25] M. Pratelli, A MiniMax Theorem without Compactness Hypothesis. Mediterr. J. of Mathematics, 2 (2005), 103-112.

[26] G. Puccetti and M. Scarsini, Multivariate comonotonicity. Journal of Multivariate Analysis, 101 (2010), 291-304.

[27] K. P. S. B. Rao and M. B. Rao, Theory of charges: a study of finitely additive measures, Academic Press, London, 1983.

[28] R. T. Rockafellar, Convex Analysis, Princeton University Press, Princeton, 1972.

[29] L. Ruschendorf and S. Kiesel, On optimal allocation of risk vectors. Insurance: Mathematics and Economics 47 (2010), 167-175.

[30] W. Schachermayer, The Fundamental Theorem of Asset Pricing under Proportional Transaction Costs in Finite discrete Time, Mathematical Finance 14 (2004), No. 1, 19-48. 